Mechanical form-Finding of the Timber Fabric Structures with Dynamic Relaxation Method

by

Seyed Sina Nabaei, Olivier Baverel and Yves Weinand

Reprinted from

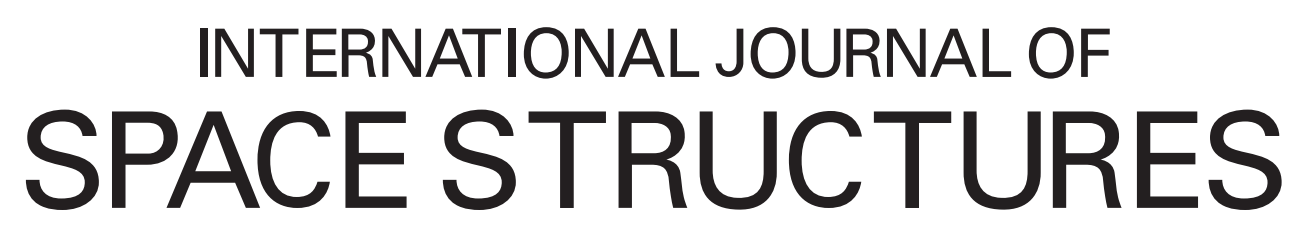

Volume $28 \cdot$ Number $3 \& 4 \cdot 2013$

MULTI-SCIENCE PUBLISHING CO. LTD. 5 Wates Way, Brentwood, Essex CM15 9TB, United Kingdom 


\title{
Mechanical form-Finding of the Timber Fabric Structures with Dynamic Relaxation Method
}

\author{
Seyed Sina Nabae ${ }^{a}, *$, Olivier Baverel ${ }^{b}$ and Yves Weinand ${ }^{a}$
}

\author{
${ }^{a}$ Chaire of Timber Construction (IBOIS), École Polyethnique Fédérale de Lausanne (EPFL), Lausanne, Switzerland \\ bNavier research unit, École des Ponts ParisTech (ENPC), Champes-Sur-Marne, France
}

(Submitted on 01/06/2013, Reception of revised paper 14/07/2013, Accepted on 26/09/2013)

\begin{abstract}
Timber Fabric structures (TFS) initiate from a correspondence between textile principles and recent industrial developments in producing cross laminated timber panels. Several individual timber strips are interlaced according to a pattern and result in an innovative space structure. The obtained three-dimensional geometry can be regarded as the relaxed configuration of deformed panels under the imposed boundary conditions. We herein propose a form-finding procedure, which reproduces this deformed configuration as the steady state of a pseudo transient constrained dynamic problem. The corresponding nonlinear problem involves finite rotation regime and contact handling through the cross section and on both panel faces. To effectively deal with nonlinear constraints, a new modified dynamic relaxation method is herein used which combines elastic material behavior with a fictitious stiffness proportional damping into an equivalent fictitious viscous material model. The procedure is implemented as an ABAQUS/Explicit user subroutine VUMAT and the overall accuracy of the numerical results has been studied for a number of geometrically nonlinear shell benchmark problems. This numerical approach is then employed to simulate the assembly process for a Timber Fabric Module (TFM), an interlaced assembly of two timber strips. The simulated geometry for the deformed surfaces is then extracted and is compared with a 3D processed surface mesh obtained from scanning a built-in prototype with noncontact Laser scanner arm to validate the simulation procedure.
\end{abstract}

Key Words: dynamic relaxation, dynamic explicit, timber fabric structures, finite elements method, 3D mesh processing

\section{INTRODUCTION}

\subsection{Background}

Timber Fabric Module (TFM) (Fig 1.b) originates from a research project at EPFL-IBOIS on applying textile principals at building scale, in the particular context of timber structures. [1,2] This new concept structures comes from an analogy between fabric production techniques (weaving, braiding, knitting, etc.), the micro scale structure of textiles and lightweight architecture using Timber panels.
The common denominator of different interlacing techniques is indeed a knot. (See Fig 1.a) The key to the approach employed in Timber Fabric project is to give a particular interpretation of the "knot" by replacing yarns with panels to reproduce a more complex overlap. (Fig 1.b) Generalization of the weaving concept is straightforward: weaving further with two strips leads to a braided arch. (See Fig 1.c with three parallel braided arches)

This two-strand braided arch can then be spatially multiplied both in depth, as double layer braided 
Woven fabric

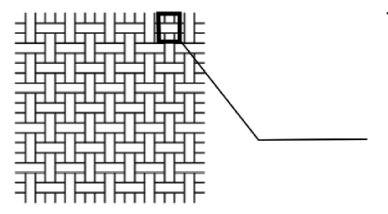

Unit cell

(a)

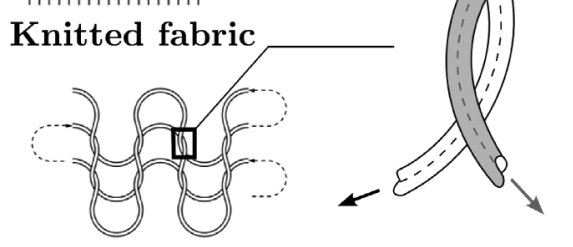

(b)
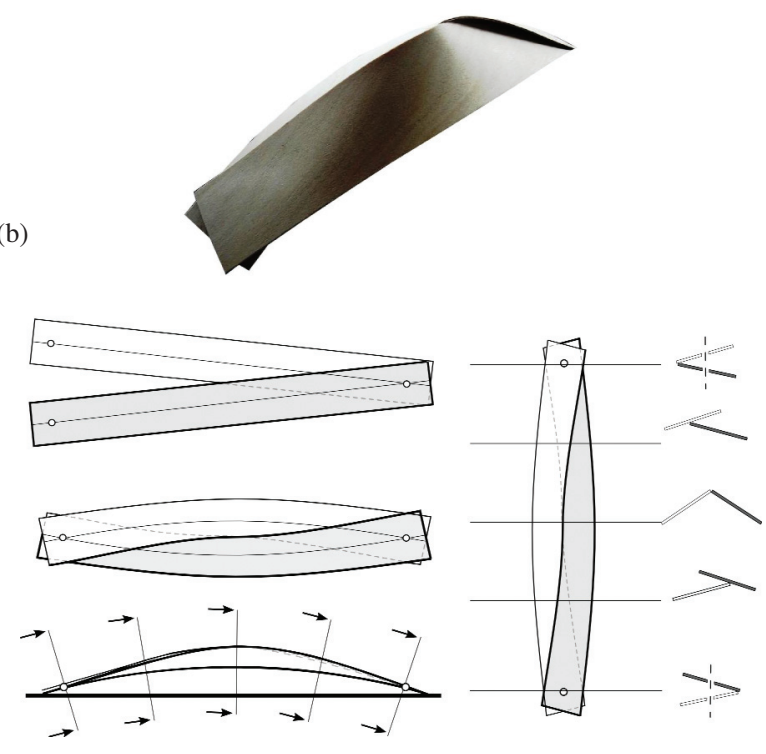

(d)

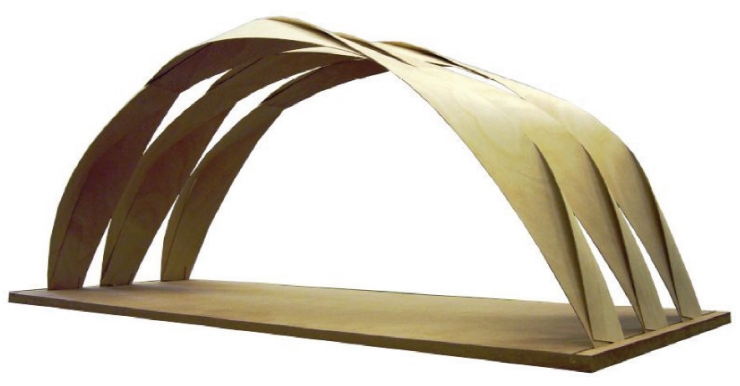

Figure 1. (a) The unit cell of textile assemblies: a two strand knot (b) Timber Fabric Module prototype and assembly process (c) Timber Fabric Structure: three parallel two strand braided arches.

arches, and in transversal direction, with multiple parallel arches connected together. [1] He proposes different double-layering and transversal multiplication techniques based on an insight on the local connection technology and the structural performance. Double layered braided arches have different weaving pattern for top and bottom layers to improve structural performance and recover the weak spots. Hudert [1] has also examined connection pieces which integrate transversal with lateral load patching and conducted prototype fabrication in medium and large scales. His morphological investigation has mainly been based on a heuristic approach with prototypes in growing scales. The prototyping approach, although is helpful to understand and resolve conceptual issues (mainly related to local assembly aspects) limits the discovered morphology to the designer's creativity and his hand making capabilities.

Once regarded through a farther point of view, the Fabric structure concept lays in an interdisciplinary context between topology/knot theory, structural engineering and wood technology. In such a context, further conceptual structures can be introduced with multithread braids (not only limited to two as in [1]) and with more populated overlaps. However, these complex structures pass quickly out of reach of a heuristic approach and the remaining conceptual potential indicates the need for a form finding tool/procedure.

\subsection{Mechanical form-finding}

Regarding the structural behavior of TFS, first attempts has been made by Sistaninia et al. [3] in order to propose a simulation procedure for the TFM formfinding problem, through a nonlinear static analysis. In order to validate the simulation, their experiment proceeds with two vertical uniformly distributed loads applied on the mid-span and measure the deformation over a number marked points on panels to compare with the simulation results. The main drawbacks of the approach proceeded in [3] is that the edge-edge contact is not treated in the static nonlinear implicit solver employed and the boundary condition at the mid-contact point is a simple symmetry condition, imposed to keep panels "connected" (and not collided) at this particular point. As a consequence we are limited only to reproduce interlaced shapes for which we suppose the exact position of edge-edge contact points from a previous heuristic experiment with the prototypes. Furthermore the position of this edge-edge contact point is supposed to be given and has not been treated as an unknown which limits the validity of the approach.

Herein we propose two main contributions to TFS form-finding problem:

- Reformulating the TFS form-finding problem:

The deformed state of the Timber Fabric structures can be regarded as the relaxed configuration of a flat initial state under a set of imposed boundary conditions. These constraints can be either i) the order of panels or their offset 
at a particular overlap or ii) an imposed displacement/rotation on a degree of freedom for a structural node. Interlacing and then connecting panels together, makes them deform and initiates a pre-stressed module with active bending behavior. We postulate that knowing the flat configuration and the overlaps interpreted as mechanically meaningful $\mathrm{BCs}$ suffices to numerically calculate the relaxed form of TSF.

In order to distinguish different interlacing patterns, a new graph representation is introduced. Inspired form the 2D knot graphs in knot theory, it helps to distinguish strands and also takes into account a notation to represent the order of overlapping strands in a particular interlace node. Each strand/panel is represented with a 2D smooth curve and at each overlap the undergoing strands are disconnected locally, the continuous curve passes on top of the others in this particular overlap node. Each strand is numbered with positive integers starting from 1 , once where they start and once where they end. For intermediate nodes, the order of overlapping strands is noted using the $>$ notation: $1>2>3$ stands for a threestrand overlap where strand 1 goes on top of the strand 2 and strand 3 passes underneath. Some instances of this structural typology with more complex nodes are illustrated along with their corresponding graph representation in Fig 2. The 2D graph enables to effectively describe and discuss a braided configuration in an abstract topological representation, regardless of its dual 3D relaxed shape. The first step toward a numerical approach to study TFS morphologies is to be able to differentiate them.

- Use of Explicit procedure to effectively deal with complex edge-edge contact handling:

Once considered as a mechanical simulation, the interlacing/weaving of panels involves shell nonlinear mechanics in finite deformation. The appropriate Finite Element framework and the nonlinear analysis method should be determined in order to proceed with the simulation. The complex assembly of the panels makes us rather use an existing Finite Element package.

In terms of the numerical method, Pseudotransient explicit dynamic analysis has been efficiently used to find static solution for complex nonlinear static shell problems. In such an analysis, the initial static problem is considered as a pseudo-transient dynamic one by adding fictitious dissipative features such as damping and mass scaling. The static solution will be the steady state of the equivalent pseudodynamic problem.

However, evaluating fictitious energy dissipative parameters is a demanding task because different parameters are usually interrelated and make the manual calibration difficult. The main goal of this study is to establish a valid simulation procedure which can handle complex modeling requirement of such braided structures and at the same time enhance an automatically adapted evolution of fictitious dissipative parameters for a pseudo-transient analysis.

The paper is presented in the following order: in $\$ 2$ the Dynamic Relaxation method is briefly reviewed and a modified Dynamic Relaxation method is introduced in $\S 3$ where the DR method is reformulated as a fictitious viscous elastic material model. This fictitious material model is implemented as an ABAQUS user subroutine material VUMAT and results are crossed with a number of nonlinear shell benchmark problems for validation. Regarding the TFS form-finding problem, the proposed modified DR is used in $\$ 4$ to simulate an arbitrarily chosen configuration of Timber Fabric module (TFM). Deformed surfaces from simulation and prototype are processed and commented in $§ 5$.

\section{THE DYNAMIC RELAXATION METHOD}

\subsection{A review on the dynamic relaxation}

Dynamic Relaxation (DR) is a numerical iterative method to find the solution of a system of nonlinear equations. It is also reported as Pseudo transient dynamic analysis in the relevant literature. In structural mechanics, the method is used to solve the static equilibrium of the system by integrating the damped wave equation to find the steady state of the equivalent dynamic problem. The method has in fact been introduced in 1960s by Otter and Day [4] or Day [5] (see also [6,7]) and has its origins in the "second order Richardson method", developed by Frankel [8] in 1950.

Since 1970, DR has been exploited in many engineering problems: nonlinear analysis of plates and shells [9-13], buckling and post buckling analysis [14-16], membrane and cable net form-finding and analysis [17-19], form-finding and analysis of tensegrity structures [20], inflatable structure [21], reciprocal frame structures [22] and medical applications [23] among others. Several publications 
(a)

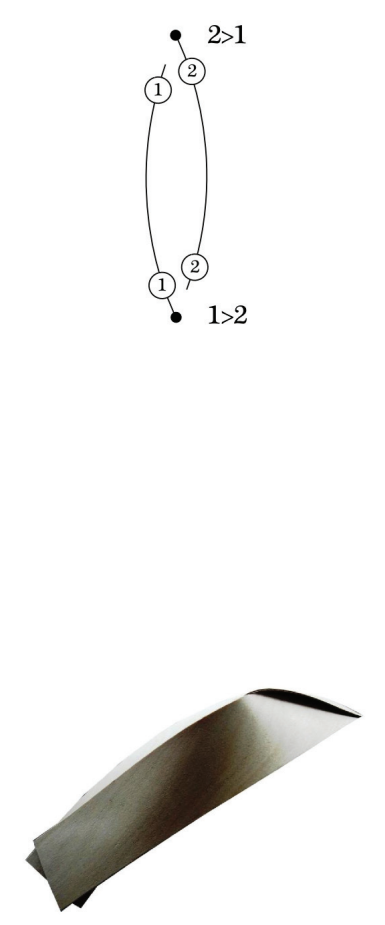

(d)
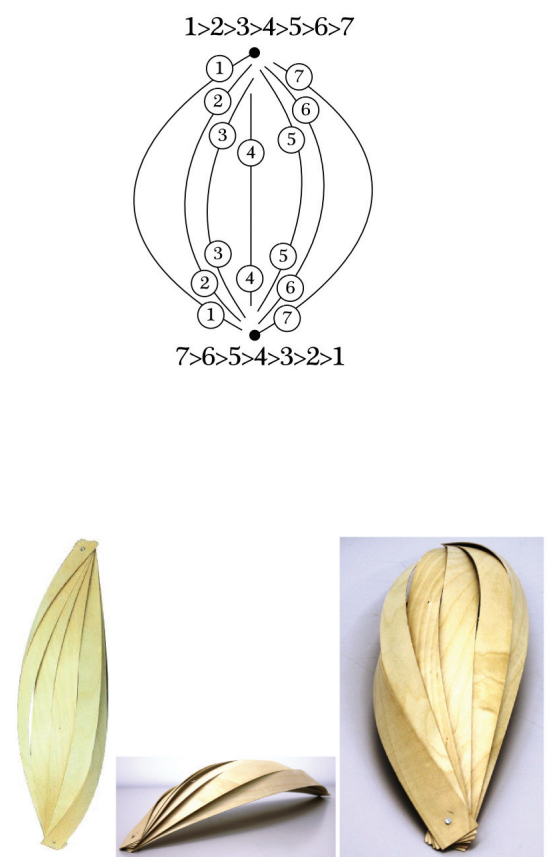

(b)

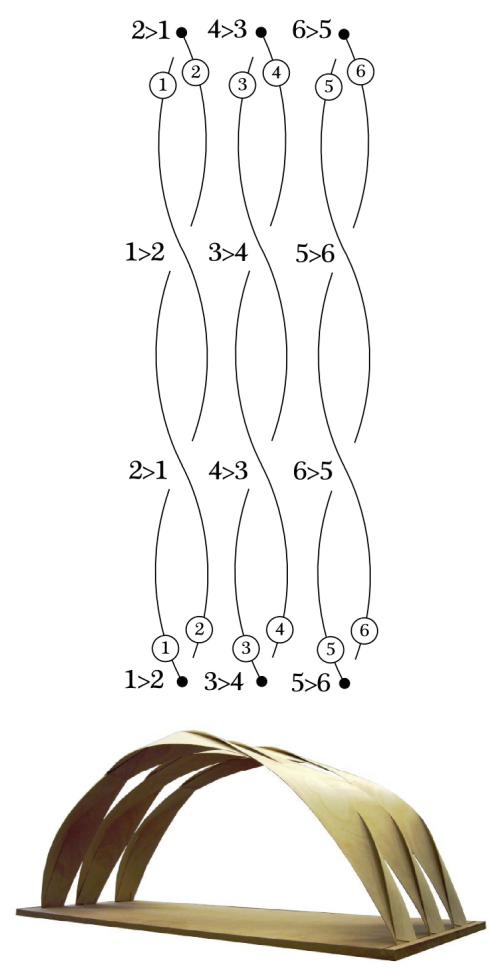

(e)

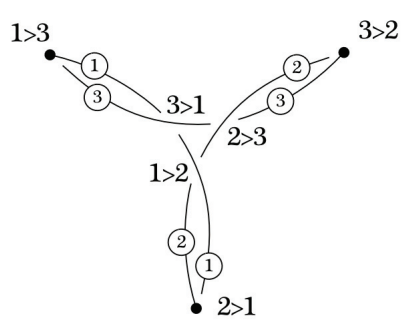

(c)
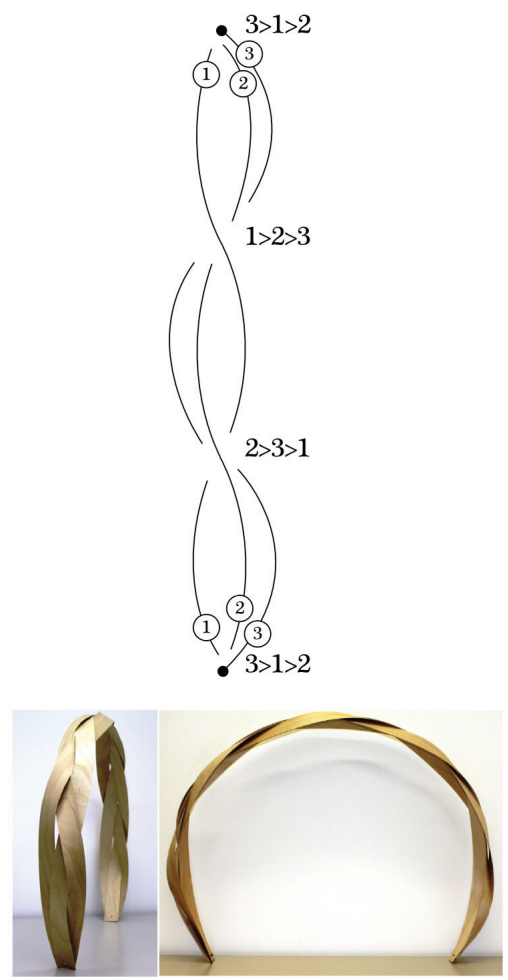

(f)
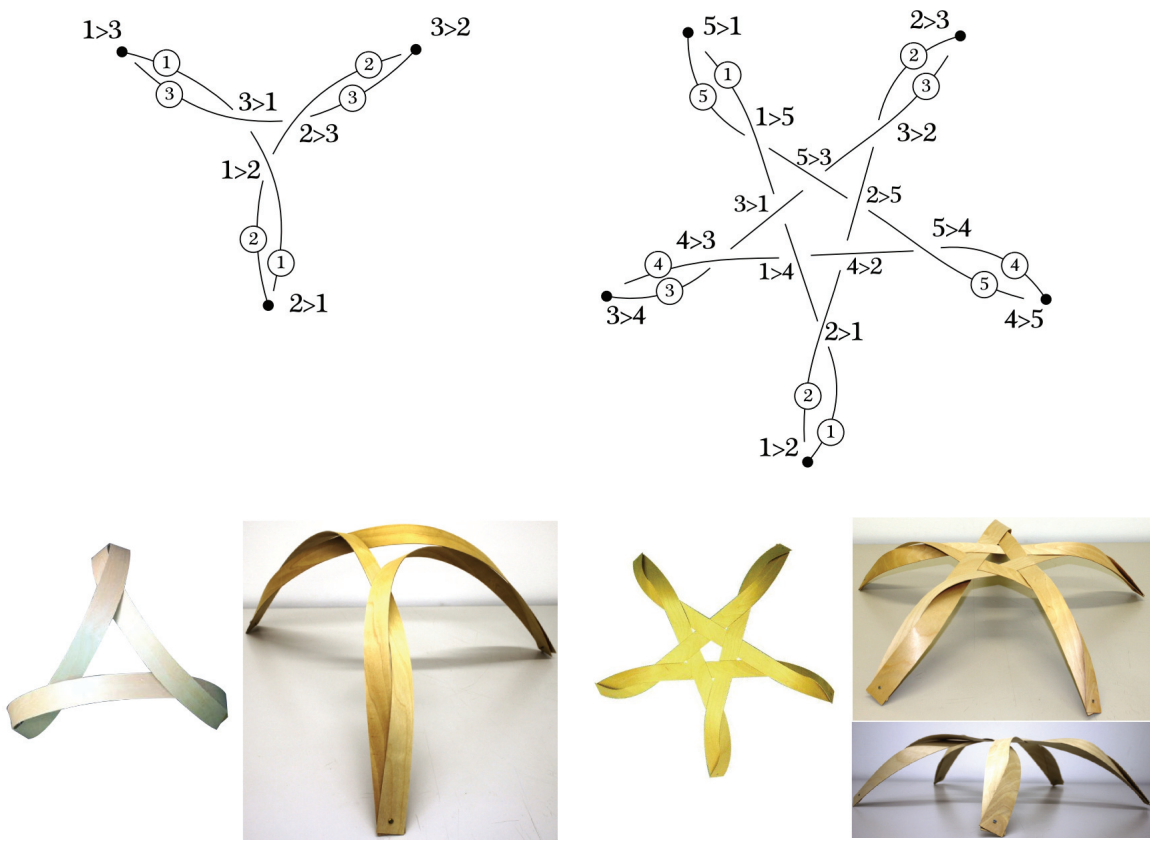

Figure 2. 2D representation of TFS: (a) A simple two-strand knot, TFM (b) Two strand braid: Three braids put side by side (c) Three strand braid (d) Multi-strand knot (e) three strand fan (f) five strand fan.

in addition, studied the convergence and the stability of the method and proposed accelerated rates thought improved viscous damping matrix, improved conditions for set up the fictitious mass matrix or introducing the Kinetic Damping. [24- 35]
The governing discretized equation for structural static equilibrium can be written as $\mathbf{K x}=\mathbf{F}$, where the solution is $\mathbf{x}^{*}=\mathbf{K}^{-1} \mathbf{F}$. In material/geometrically nonlinear problems the estimation of the tangent stiffness matrix $\mathbf{K}$ can be computationally expensive. 
In order to obtain the static solution using the DR method, fictitious mass and damping is introduced and the equivalent dynamic equation is integrated using the central difference explicit technique. Let the incremental displacement vector be $\mathbf{x}^{n}=\mathbf{x}\left(t_{n}\right)$, the dynamic equilibrium equation at $n^{\text {th }}$ time step will be

$$
\mathbf{M} \ddot{\mathbf{x}}^{n}+\mathbf{C} \dot{\mathbf{x}}^{n}+\mathbf{K} \mathbf{x}^{n}=\mathbf{F}^{n}
$$

According to the central difference integration and supposing a constant time step, mid-step velocity vector, step velocity vector and step acceleration vector can be written as Eq. 2 .

$$
\begin{aligned}
& \dot{\mathbf{x}}^{n+\frac{1}{2}}=\frac{1}{\Delta t}\left(\mathbf{x}^{n}-\mathbf{x}^{n-1}\right), \dot{\mathbf{x}}^{n}=\frac{1}{2}\left(\dot{\mathbf{x}}^{n+\frac{1}{2}}+\dot{\mathbf{x}}^{n-\frac{1}{2}}\right) \\
& \ddot{\mathbf{x}}^{n}=\frac{1}{\Delta t}\left(\dot{\mathbf{x}}^{n+\frac{1}{2}}-\dot{\mathbf{x}}^{n-\frac{1}{2}}\right)
\end{aligned}
$$

Substituting Eq. 2 values into Eq. 1, gives an iterative linearized equation for the mid step velocity vector. (see Eq. 3)

The DR algorithm can then be resumed in Alg. 1.

$$
\begin{aligned}
& \dot{\mathbf{x}}^{n+\frac{1}{2}}=\left(\frac{\mathbf{M}}{\Delta t}+\frac{\mathbf{C}}{2}\right)^{-1}\left(\left(\frac{\mathbf{M}}{\Delta t}-\frac{\mathbf{C}}{2}\right) \dot{\mathbf{x}}^{n-\frac{1}{2}}+\mathbf{F}^{n}-\mathbf{K} \mathbf{x}^{n}\right) \\
& \mathbf{x}^{n+1}=\mathrm{x}^{n}+\dot{\mathbf{x}}^{n+\frac{1}{2}} \Delta t
\end{aligned}
$$

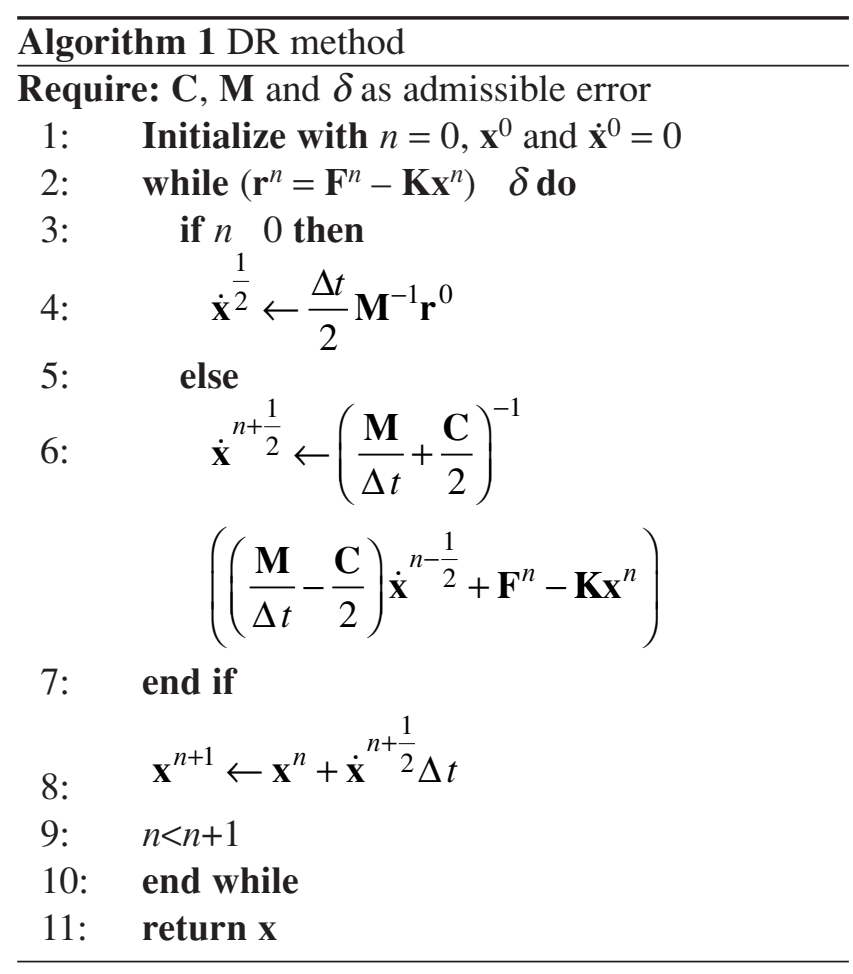

\subsection{Fictitious mass and damping}

Different choices for the damping $\mathbf{C}$ and mass $\mathbf{M}$ matrices, lead to distinct DR-derived variants where the rates of convergence and stability conditions will be different. The mass matrix $\mathbf{M}$ is usually scaled in order to increase the time-step size, although it can also be left as a lumped mass diagonal matrix. Some examples for the fictitious mass proposed in the literature are as following: a mass matrix computed from different directional densities [36], a diagonal mass matrix consisted of the diagonal elements of the stiffness matrix [26], mass matrix based on the stiffness matrix proposed with an insight on the Gerschgörin theorem [27], a mass scale factor determined based on the incremental rate of change of the kinetic energy upon internal energy for the structure [37] and finally a diagonal lumped mass matrix which is scaled at each node to align maximum eigenvalues of elements. [23] A usual approximation for $\mathbf{C}$ is the Rayleigh damping which supposes the damping to be a linear combination of mass and stiffness matrices, $\mathbf{C}=c \mathbf{M}+\kappa \mathbf{K}$. The other main alternative is to introduce the Kinetic Damping, introduced by Cassell et al. [38]. The idea behind the Kinetic Damping is to observe the evolution of the kinetic energy of the system and to reduce velocity components to zero as soon as a local kinetic energy peak is observed. Regarding the Rayleigh damping, the main approach has been to suppose a particular construction for damping matrix $\mathrm{C}$ and then to adapt the damping coefficient to a critical damping value at each iteration via the eigenvalue analysis of the iterative displacement error vector. (similar to $[39,26]$ followed in several successor publications) This approach is also called Adaptive damping in the literature.

\section{MODIFIED DYNAMIC RELAXATION METHOD}

\subsection{Mathematical formulation}

The idea here is to add the fictitious damping contribution to the material elastic response and treat the Dynamic Relaxation concept as a fictitious viscous material model. Revisiting the Rayleigh damping equation, $\mathbf{C}=c \mathbf{M}+\kappa \mathbf{K}$, a stiffness proportional damping matrix is supposed as $\mathbf{C}=\kappa \mathbf{K}$ and following the eigenvalue analysis of the error vector, a near critical damping coefficient is obtained.

Let $\mathbf{M}$ be a diagonal lumped mass matrix with diagonal elements $m_{i i}, \mathbf{D}=\mathbf{M}^{-1} \mathbf{K}$ and $\mathbf{C}=\kappa \mathbf{K}$. Substituting these into Eq. 3 and rewriting the whole regarding to the step displacement vector $\mathbf{x}$, we get Eq. 4. 


$$
\begin{aligned}
& \boldsymbol{\alpha}_{1} \mathbf{x}^{n+1}+\boldsymbol{\alpha}_{2} \mathbf{x}^{n}+\boldsymbol{\alpha}_{3} \mathbf{x}^{n-1}-\mathbf{F}^{n}=0 \\
& \boldsymbol{\alpha}_{1}=\frac{\mathbf{M}}{\Delta t^{2}}\left(\mathbf{I}+\frac{\kappa \Delta t}{2} \mathbf{D}\right) \\
& \boldsymbol{\alpha}_{2}=\frac{\mathbf{M}}{\Delta t^{2}}\left(\Delta t^{2} \mathbf{D}-2 \mathbf{I}\right) \\
& \boldsymbol{\alpha}_{3}=\frac{\mathbf{M}}{\Delta t^{2}}\left(\mathbf{I}-\frac{\kappa \Delta t}{2} \mathbf{D}\right)
\end{aligned}
$$

Following [39] and [26], if $\mathbf{x}^{*}$ is the solution for the static equilibrium equation $\mathbf{K x}=\mathbf{F}$, then let's introduce the incremental displacement error vector as $\varepsilon^{n}=\mathbf{x}^{n}-\mathbf{x}^{*}$. Furthermore, successive error vectors are supposed to be linearly dependent via a matrix $\mathbf{E}$ as $\varepsilon^{n+1}=\mathbf{E} \varepsilon^{n}$. Let $\lambda_{E}$ be the largest eigenvalue of $\mathbf{E}$ (including the nonReal ones), then $\varepsilon^{n+1}=\lambda_{E} \varepsilon^{n}=\lambda_{E}^{2} \varepsilon^{n-1}$. In order to have the iterative method converge, $\lambda_{E}$ should have its complex norm less than one, $\lambda_{E}<1$. Substituting incremental error vector into Eq. $4, \lambda_{E}$ can be calculated as the solution of a quadratic equation, where $m, \lambda_{D}$ are respectively any eigenvalue of $\mathbf{M}$ and $\mathbf{D}$.

$$
\begin{aligned}
\lambda_{1} \lambda_{E}^{2} & +\lambda_{2} \lambda_{E}+\lambda_{3}=0 \Rightarrow \\
\lambda_{E} & =\frac{-\lambda_{2} \pm \sqrt{\lambda_{2}^{2}-4 \lambda_{1} \lambda_{3}}}{2 \lambda_{1}} \\
\lambda_{1} & =\frac{m}{\Delta t^{2}}\left(1+\frac{\kappa \Delta t}{2} \lambda_{D}\right) \\
\lambda_{2} & =\frac{m}{\Delta t^{2}}\left(\Delta t^{2} \lambda_{D}-2\right) \\
\lambda_{3} & =\frac{m}{\Delta t^{2}}\left(1-\frac{\kappa \Delta t}{2} \lambda_{D}\right)
\end{aligned}
$$

It can be shown that the minimum $\lambda_{E}$ occurs when the quadratic equation Eq. 5 has two equal real roots which correspond to a near critical damped system. (see Fig. 3)

In case of explicit time integration, maximum stable time step must satisfy the condition $\Delta t_{c r} \leq \frac{2}{\omega_{\max }}$ for an undamped systems with $\omega_{\max }$ be the maximum frequency of the system and $\Delta t_{c r} \leq \frac{2}{\omega_{\max }} \leq \frac{2}{\omega_{\max }}$ $\left(\sqrt{\xi^{2}+1}-\xi\right)$ in case of damping, where $0.0 \leq \xi \leq 1.0$ is the fraction of critical damping in the mode with the highest frequency. [40] The relation for the maximum time step size can also be written as a function of $\lambda_{\max }$ $=\omega_{\max }^{2}$, the maximum eigenvalue of the system, as $\Delta t_{c r}=\frac{2}{\sqrt{\lambda_{\max }}}$.

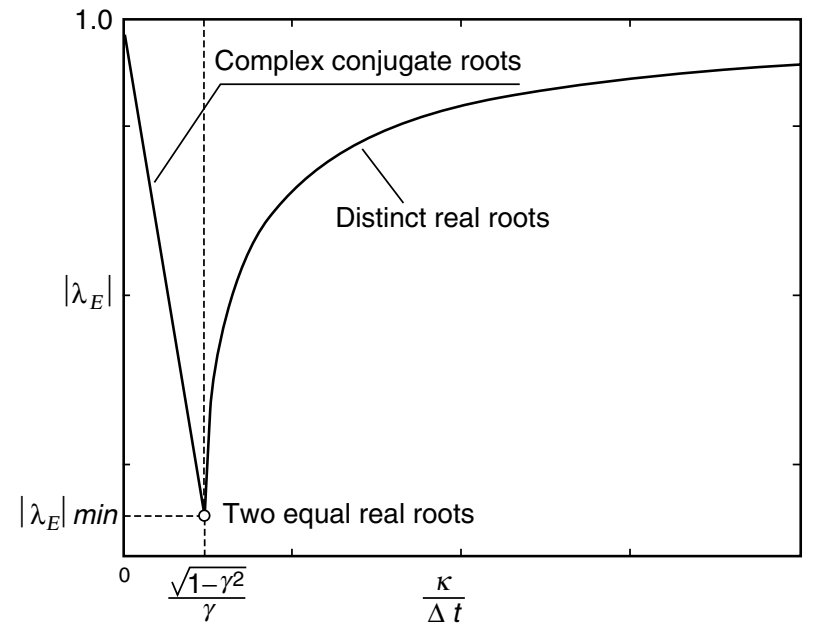

Figure 3. Relation between $\frac{\kappa}{\Delta t}$ and $\left|\lambda_{E}\right|$.

Following the notation used in Eq. 5, it is convenient to recall that $\lambda_{\max }$ is related to the maximum eigenvalue of $\mathbf{D}=\mathbf{M}^{-1} \mathbf{K},\left(\lambda_{D}\right)_{\max }[41,40]$ and since the Eq. 5 is true for any eigenvalue, one can suitably replace $\lambda_{D}$ as a function of maximum time step size $\Delta t_{c r}$. If then we suppose that the actual increment time step, $\Delta t$ is also proportional to this stable time step size by a real coefficient $0.0 \leq \gamma \leq 1.0 \in$ as $\Delta t=\gamma \Delta t_{c r}$, then all terms of the discriminant in Eq. 5 can be rewritten with respect to $\gamma, t$ and $\kappa$. Once set to zero in order to obtain the fastest convergence condition, following equation can be established between the stiffness proportional viscous damping coefficient $\kappa$ and the time step

$$
\frac{\kappa}{\Delta t}=\frac{\sqrt{1-\gamma^{2}}}{\gamma}
$$

Regarding now the discretized system, in explicit central difference FEM, the maximum eigenvalue of the assembled system $\lambda_{\max }$ is bounded with sufficient accuracy, by the maximum eigenvalue of any element $\lambda \max _{\max }^{(\mathrm{e})}$ or $\Delta t_{c r}^{(e)}=\frac{2}{\sqrt{\lambda_{\max }^{(e)}}} \leq \Delta t_{c r} \leq \frac{2}{\sqrt{\lambda_{\max }}}$, where $\Delta t_{c r}^{(e)}$ is the element's stable time step size. [41] On the other hand, the maximum stable time step for any element must satisfy the Courant-Friedrichs-Lewy condition [42], which is determined based on the smallest transit time of a dilatation wave across any of the elements in the mesh. The Courant-Friedrichs-Lewy condition in our context of structural mechanics can be resumed as $\Delta t_{c r}^{(e)} \leq \frac{L^{(e)}}{c} \leq \Delta t_{c r}$. Here, $L^{(e)}$ is the element characteristic length (or if simplified, the "smallest" 
dimension of the element), $\left.c=\sqrt{(} \frac{\lambda+2 \mu}{\rho}\right)$ and $\lambda, \mu$ are the Lamé constants, $\rho$ being the density.

In numerical implementation of explicit FEM solvers, estimation of system frequencies at each increment to determine the maximum stable time step size is an expensive task, so the Courant-FriedrichsLewy condition is practically verified for the smallest element in order to determine the $\Delta t_{c r}$.

This, along with the fact that we have related the stiffness proportional damping coefficient $\kappa$ to the time step size through Eq. 6, enables us to adapt the fictitious stiffness proportional damping contribution to a near critical value at each time step only based on the time step size estimation. In fact the ratio $\frac{\min \Delta t_{c r}^{(e)}}{\Delta t_{c r}}$ is equivalent to the coefficient $\gamma$ in Eq. 6, which should be selected deliberately by the user in our algorithm. This ratio can be calculated knowing the Finite Element's mass and stiffness matrices and the problem configuration. Finite Element's mass and stiffness matrices would give us the maximum eigenvalue of the element and the maximum stable time step

for the element is $\Delta t_{c r}^{(e)}=\frac{2}{\sqrt{\lambda_{\max }^{(e)}}}$. (see [43] for a demonstration for thin shell problems) Here we take the approximate value of $\gamma=0.9$ for our numerical simulation. The main interest of a stiffness proportional matrix over the other proposed options is that it can be implemented as a viscous elastic material model and use an existing time step estimation algorithm inside an explicit procedure, without explicitly having access to assembled mass and stiffness matrices.

The viscous stress is generated proportional to the strain rate in form of $\sigma^{\text {vis. }}=\kappa \mathbb{C} \dot{\varepsilon}$. The total damped stress $\sigma=\mathbb{C} \varepsilon+\kappa \mathbb{C} \dot{\varepsilon}$ is then used to evaluate the amount of internal force, necessary to compute the accelerations for velocity update. The abovementioned idea is resumed under the following algorithm.

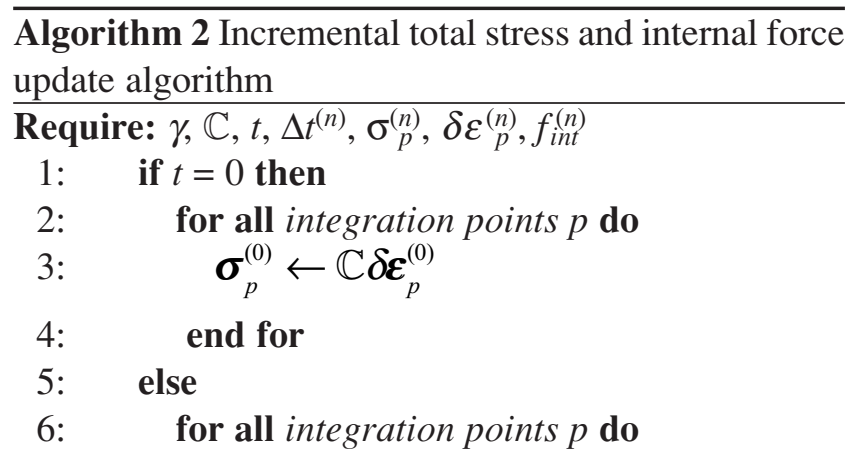

7:

8:

$$
\begin{aligned}
& \boldsymbol{\varepsilon}_{p}^{(n+1)} \leftarrow \boldsymbol{\varepsilon}_{p}^{(n)}+\delta \boldsymbol{\varepsilon}_{p}^{(n)} \\
& \boldsymbol{\sigma}_{p}^{(n+1)} \leftarrow \mathbb{C} \boldsymbol{\varepsilon}_{p}^{(n+1)}+\left(\frac{\sqrt{1-\gamma^{2}}}{\gamma}\right) \mathbb{C} \delta \boldsymbol{\varepsilon}_{p}^{(n)}
\end{aligned}
$$$$
\text { 9: } \quad f_{\text {int }}^{(n+1)} \leftarrow f_{i n t}^{(n)}+\left(\frac{\boldsymbol{\sigma}_{p}^{(n+1)}+\boldsymbol{\sigma}_{p}^{(n)}}{2}\right) \delta \boldsymbol{\varepsilon}_{p}^{(n)}
$$

10: end for

11: end if

12: $\quad$ return $\sigma_{p}^{(n+1)}, f_{\text {int }}^{(n+1)}$

$\gamma \quad$ Viscous damping parameter, $\frac{\kappa}{\Delta t}=\frac{\sqrt{1-\gamma^{2}}}{\gamma}$

$\mathbb{C} \quad$ Material stiffness matrix

$\Delta t^{(n)}$ Time step size in $n^{\text {th }}$ increment

$\sigma^{(n)} \quad$ Cauchy stress vector in $n^{\text {th }}$ time increment

$\delta \varepsilon^{(n)}$ Incremental Strain vector in $n^{\text {th }}$ increment

$\varepsilon^{(n)} \quad$ Green Strain vector in $n^{\text {th }}$ increment

$f_{\text {int }}^{(n)} \quad$ Internal force in $n^{\text {th }}$ increment

The duration of the analysis should be picked by the user with respect to the simulation problem and long enough to continue until the Kinetic energy of the system becomes stationary.

\subsection{Numerical implementation}

In this section numerical results obtained from the simulation with VUMAT user subroutine are examined for accuracy. In particular, verification is realized using results presented in [44, 45, 46, 47] among others.

Four problems are treated herein: The isotropic elastic behavior is assumed through §3.2.1 §3.2.3 and an orthotropic laminate case is studied in \$3.2.4. The case study $\$ 3.2 .1$ is a classical benchmark problem with analytical solution to test the large rotation of shells. The bending/torsional coupling is tested in \$3.2.2 and inextensible bending behavior is examined with the buckling hemisphere problem in §3.2.3. This problem is considered as both a linear and a nonlinear benchmark problem; here loads are increased until reaching the large deformation regime. Finally, the post buckling behavior of an orthotropic laminate cylindrical roof is studied in \$3.2.4 for two lamination to demonstrate the generality of the proposed approach.

The ABAQUS S4, 4-node quadrilateral shell Finite element with explicit time integration scheme and second order accuracy is used for all simulations. The VUMAT user subroutine describes the fictitious viscous material behavior and replaces the software's default elastic behavior in order to determine stress and internal energy update for each increment. According to the 
communicated syntax, VUMAT must define the Cauchy stress and the rotations are applied by the framework with the co-rotational formulation based on the Green-Naghdi rate. The rotation however should be implemented if a rate other than the default one is needed.

The analysis is run over time till it reaches a steady state for the desired degree of freedom. The only dissipative feature is the fictitious stiffness proportional damping with $\gamma=0.9$, as detailed in Alg. 2 . The default linear bulk viscosity parameter is thus turned off. The proposed approach is based on a pseudo-transient dynamic analysis, so wherever in the case studies the density is not specified, we assumed the deliberately chosen value of $500 \frac{\mathrm{kg}}{\mathrm{m}^{3}}$.

\subsubsection{Rollup a clamped plate strip}

Distributed moment is applied on one end of a plate strip while the other end is clamped. This problem has an analytical solution for the tip deflection in 2D case (in xz plan):

$$
\begin{aligned}
& u_{1}(A)=\frac{E I}{M} \sin \left(\frac{M l}{E I}\right)-l \\
& u_{3}(A)=\frac{E I}{M}\left(1-\cos \left(\frac{M l}{E I}\right)\right)
\end{aligned}
$$

If $M=M_{\max }=\frac{2 \pi E I}{l}$, the strip is rolled up into a full circle. The problem configuration is shown in fig. 4 and material properties is set as $E=1.2 \times 10^{6}, v=0.0$. The results obtained for simulations with $\frac{M}{M_{\max }}=1.0$,
$0.9,0.8,0.6,0.4,0.3,0.2,0.15,0.1$ is compared with the computed analytical solution and deformed configuration are also extracted for a number of the load cases in Fig. 5.

\subsubsection{Annular ring plate under transversal shear edge force}

An isotropic open ring is clamped on one edge and a uniformly distributed edge shear force $\mathrm{P}$ is applied to the other edge along the $\mathrm{z}$ axis. (see Fig. 6) The strip undergoes a spring like oscillation under bending and torsion caused by the sheared edge force until it reaches the equilibrium steady state. The plate dimensions are specified in Fig. 6 and the thickness is $h=0.03$. Material elastic properties are $E=21 \times 10^{6}$, $v=0.0$. The strip is simulated under $\frac{P}{P_{\max }}=0.1,0.2$, $0.4,0.5,0.6,0.8,0.9,1.0$, load cases and vertical deflection of points A and B (two ends of the loaded edge) is extracted to plot the load-deflection curve.

The final load case $\left(P=P_{\max }=0.8\right)$ involves a deformation of $\approx 3 \times r_{i}$. Deformed configurations are plotted without magnification in Fig. 5.a and the simulation load-deflection curve for both end points are compared with results presented in $[44,45]$ among others.

\subsubsection{Buckling of a pinched hemisphere}

Two equal point loads $P$ are applied to a pinched hemispherical shell with a hole on top. The problem is simulated in one quadrant thanks to the symmetry boundary conditions at opposite ends. (See Fig. 8)

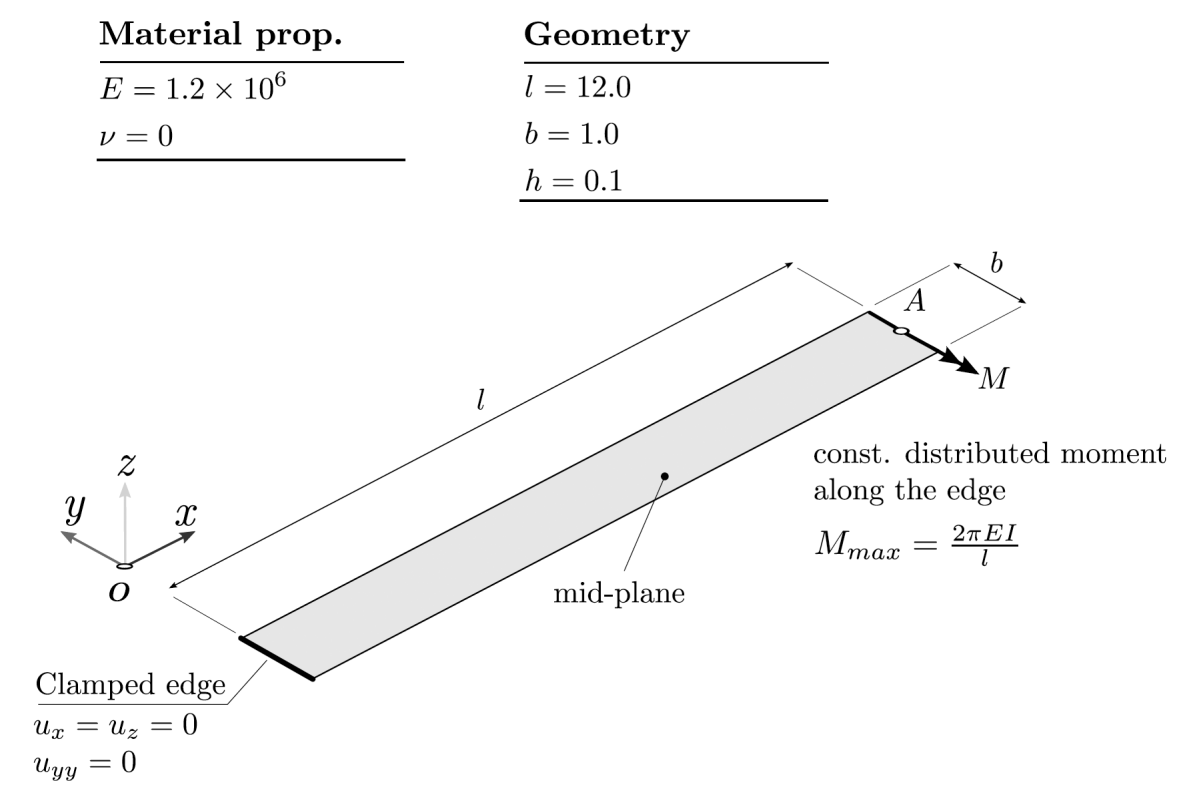

Figure 4. Clamped strip problem $\$ 3.2 .1$. 
(a)

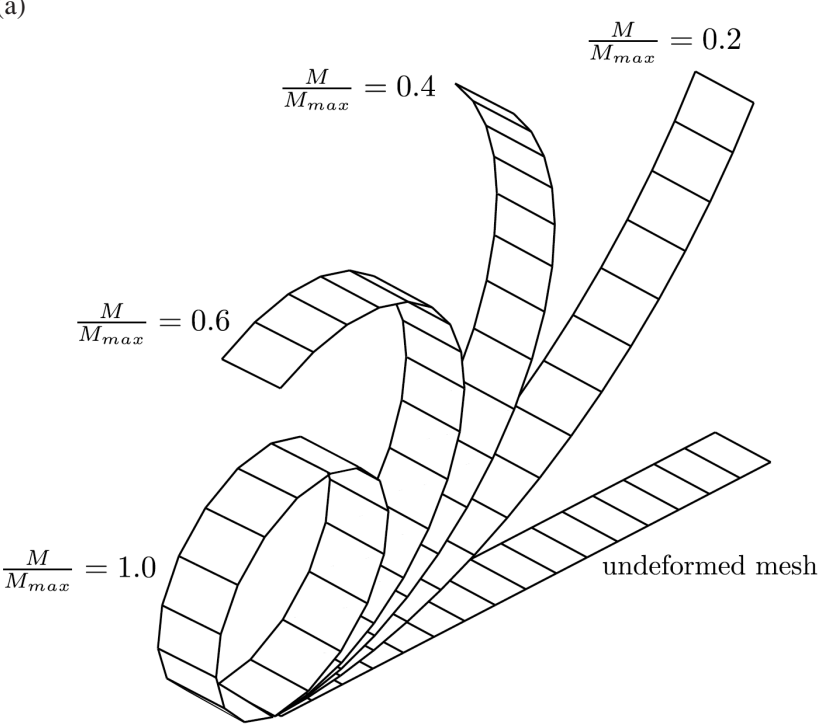

(b)

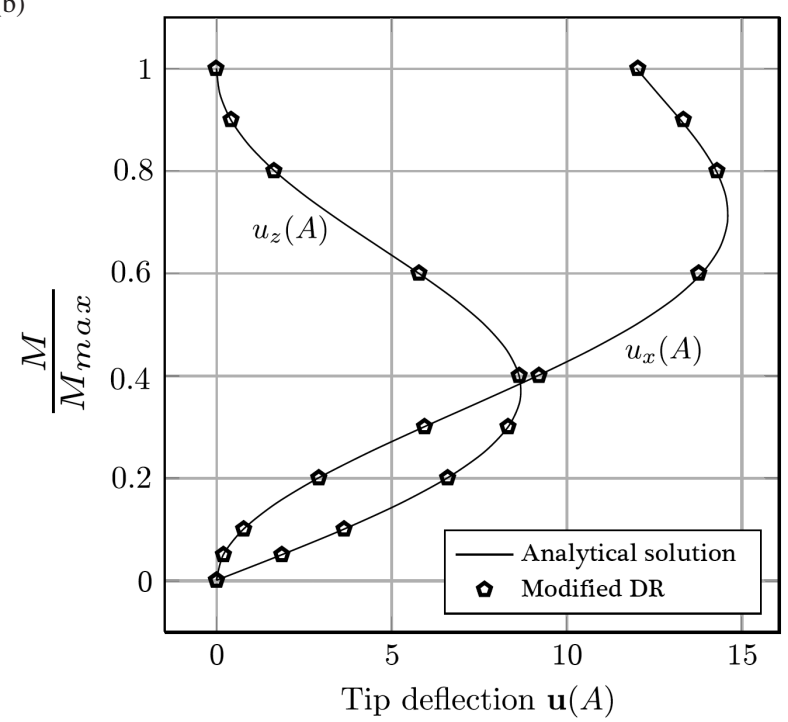

Figure 5. Clamped strip simulation results: (a) Deformed mesh for loadcases (b) Tip deflection $u_{x}(A), u_{z}(A)$ versus end moment ratio $\frac{M}{M_{\max }}$.

\begin{tabular}{l} 
Material prop. \\
\hline$E=21.0 \times 10^{6}$ \\
$\nu=0.0$
\end{tabular}

Clamped edge $u_{x}=u_{y}=u_{z}=0$ $u_{x x}=u_{y y}=u_{z z}=0$

\section{Geometry}

$r_{i}=6.0$

$r_{e}=10.0$

$h=0.03$

const. distributed force

along the edge $A B$

$P_{\text {max }}=0.8$
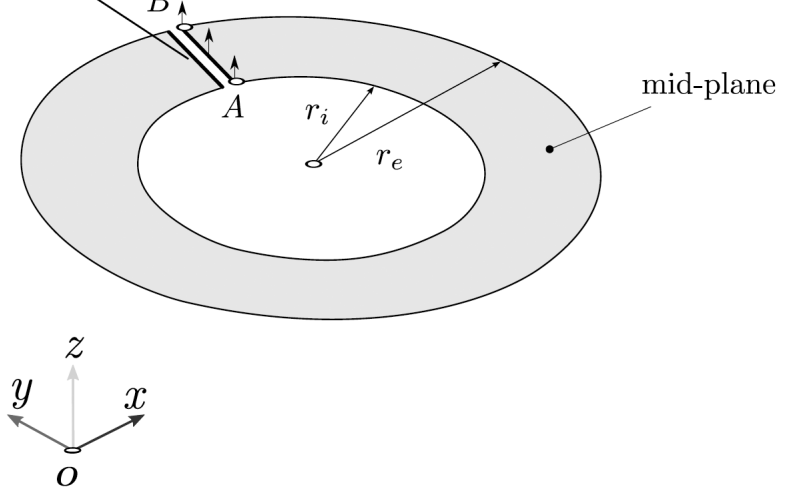

Figure 6. Annular ring problem §3.2.2.

This problem is a test to examine the method's ability to represent inextensible bending behavior and rigid body modes and is used as both linear and nonlinear benchmark, depending on the applied load level. Here the load is increased to cause a deformation level up to $60 \%$ of the radius to be placed in a large deformation regime. The material is assumed to be linear elastic with $E=6.825 \times 10^{7}, v=0.3$ and shell thickness is $h=0.04$.
Radial deflections under the applied load curves are extracted for load cases $P=20,40,60,80,100$, $120,140,160,180,200$, and are compared to transcribed results in the literature. Particularly, Modified DR results agree with values ratified by Simo et al. [47] which are also reported by Buechter and Ramm [46], Jiang and Chernuka [48]. These curves are meanwhile different with values which appear in [44]. (See Fig. 9.b) 
(a)

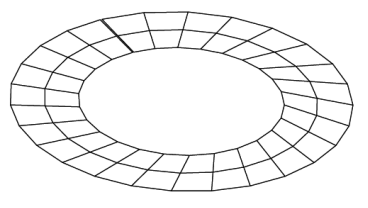

undeformed mesh

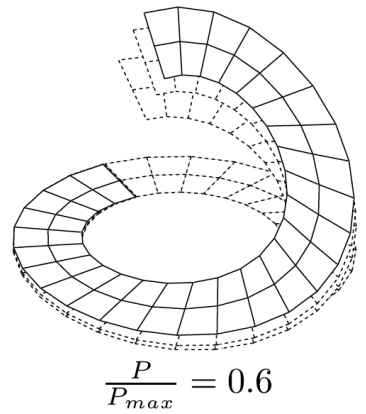

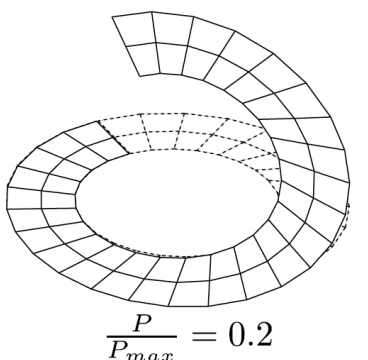

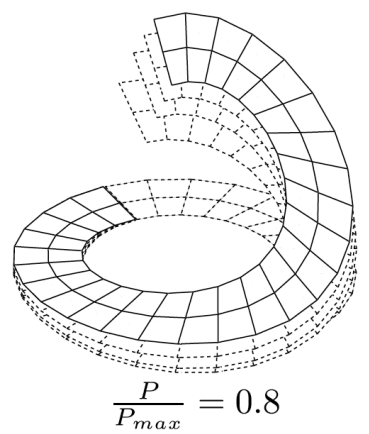

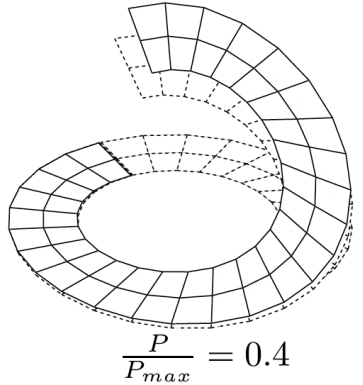

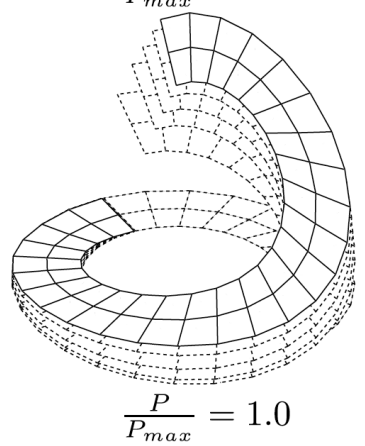

(b)

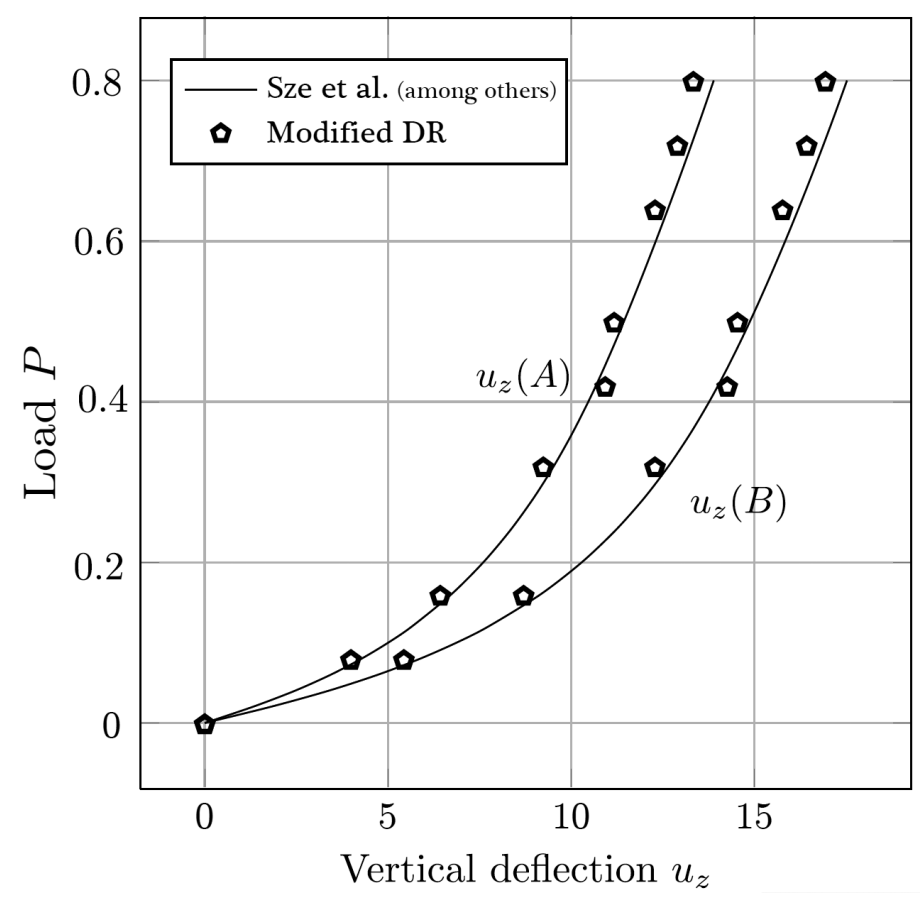

Figure 7. Annular ring plate: (a) Superimposed deformed mesh for load cases (b) vertical deflection $u_{z}$ versus free edge shear force $\mathrm{P}$.

3.2.4. Hinged cylinder roof: post-buckling behavior of an orthotropic laminate

A vertical point load $\mathrm{P}$ is applied to the midpoint of a cylindrical shell with hinged boundary condition assumed for lateral edges. The other two transversal curved edges are supposed to be free. The problem involves buckling and post-buckling of a lightly curved laminate shell. (see Fig. 10 for problem configuration)

Two laminate configurations are considered: $\left[0^{\circ} / 90^{\circ} / 0^{\circ}\right]$ and $\left[90^{\circ} / 0^{\circ} / 90^{\circ}\right]$. All lamination plies have the same equal thickness of $\frac{h}{3}$ and ply material properties is assumed as $E_{L}=3300, E_{T}=1100, G_{L T}=$ $660, v_{L T}=v_{T T}=0.25$. Ply direction $0^{\circ}$ is once the 


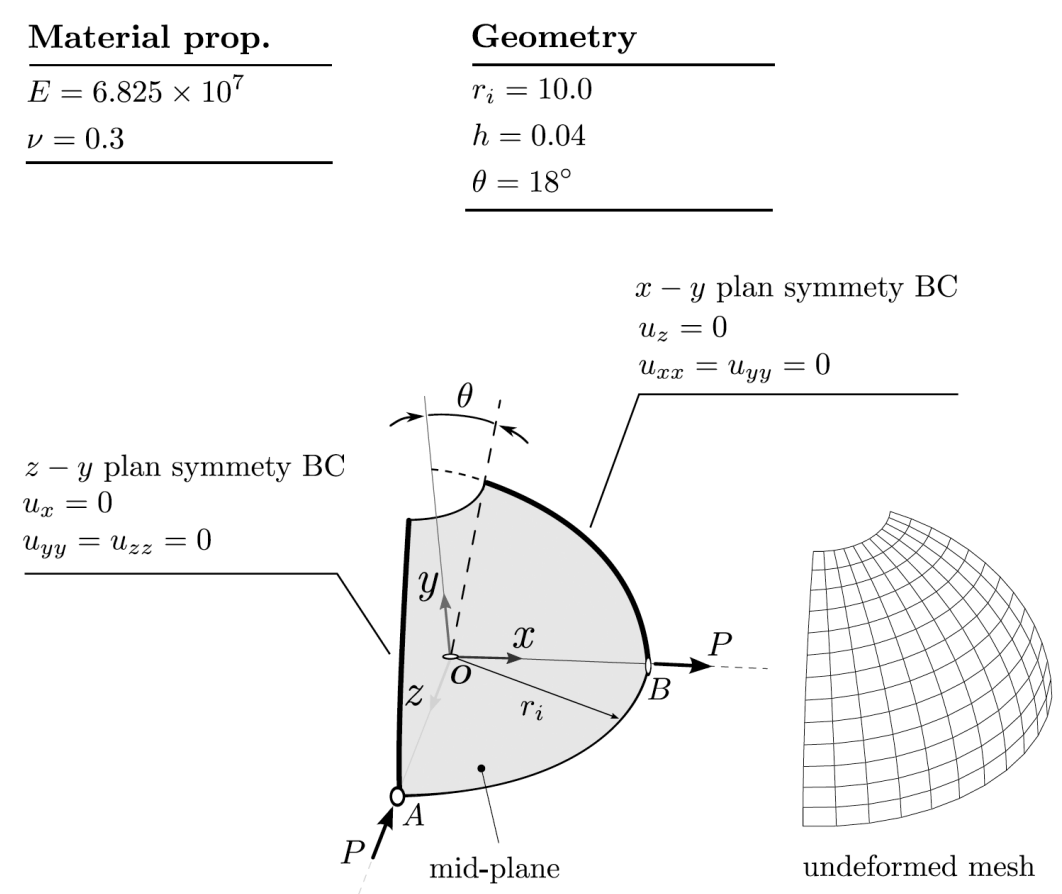

Figure 8. Buckling of a pinched hemisphere problem §3.2.3.

(a)

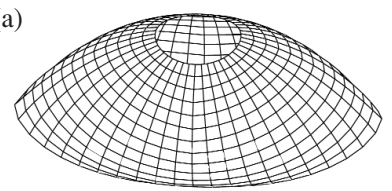

$P=100$

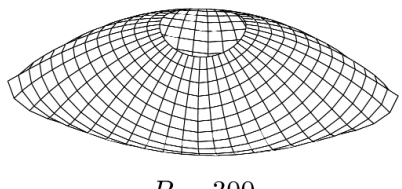

$P=300$

(b)

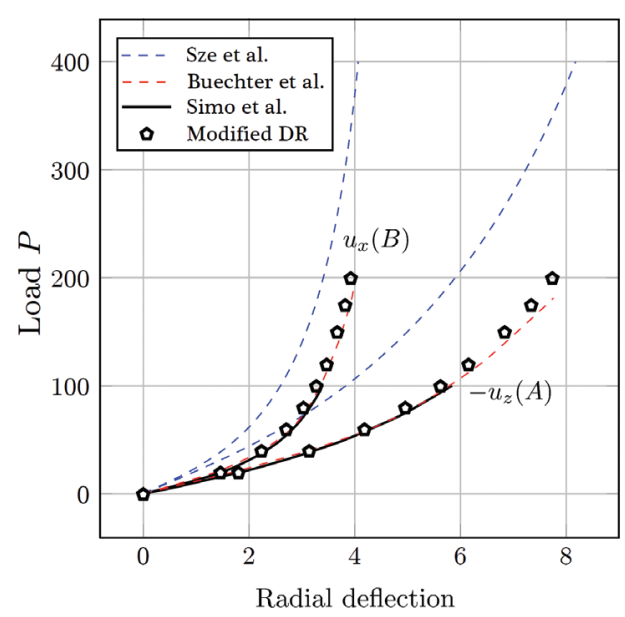

Figure 9. Buckling of a pinched hemisphere: (a) deformed mesh configuration for $\mathrm{P}=100 ; 200 ; 300 ; 400$ (b) Radial deflection versus radial load $\mathrm{P}$.

fibers are along the longitudinal edge. Viscous material definition and can be used with both imposed load and/or imposed displacement regimes. Here the problem is simulated with imposition of the various displacement levels at the midpoint: instead of the point load, the vertical displacement is imposed at that point and vertical reaction force is extracted. (once steady state is reached for the reaction force). The load-deflection curve is extracted for the midpoint displacement $-u_{z}(C)=2.5,5,10,12.5,15,17.5,20$, $23,25,27.5,30,31.66$, and is compared with the results reported by Sze et al. [44] and Arciniega and Reddy [45] among others. (See Fig. 11)

\section{TFM PROTOTYPE SIMULATION 4.1. Prototype geometry and assembly process}

A medium scale prototype has been built from laminated wood panels of size $2340 \mathrm{~mm} \times 240 \mathrm{~mm} \times$ $6.7 \mathrm{~mm}$ to verify simulation results. (see Fig. 12) In order to ensure that relative panel offset and spatial angles are respected at their correspondent connection, three wooden wedges per connection point are exploited for this purpose. The module is fixed with three bolt per wedge connection into a rigid. The set of three wedges at one end is mirrored of the other set, corresponding to the mid-plan normal to the y axis. The geometrical parameters for the prototype are as following (notations illustrated in Fig. 14).

$\begin{array}{llll}l & 2060 \mathrm{~mm} & \theta_{x 1} & 17^{\circ} \\ w & 240 \mathrm{~mm} & \theta_{x 2} & 11.5^{\circ} \\ d & 47.7 \mathrm{~mm} & \theta_{y 1} & 14.5^{\circ} \\ d^{\prime} & 76 \mathrm{~mm} & \theta_{y 2} & -8^{\circ}\end{array}$




\begin{tabular}{llll} 
Material prop. & Geometry & & Load \\
\hline$E_{L}=3300$ & $\frac{r_{i}=2540}{P_{\max }=3000}$ \\
$E_{T}=1100$ & $l=2 \times 254$ & \\
$\nu_{L T}=\nu_{T T}=0.25$ & $h=12.7$ & \\
$G_{L T}=660$ & $\theta=0.1 \mathrm{Rad}$ & \\
\hline
\end{tabular}

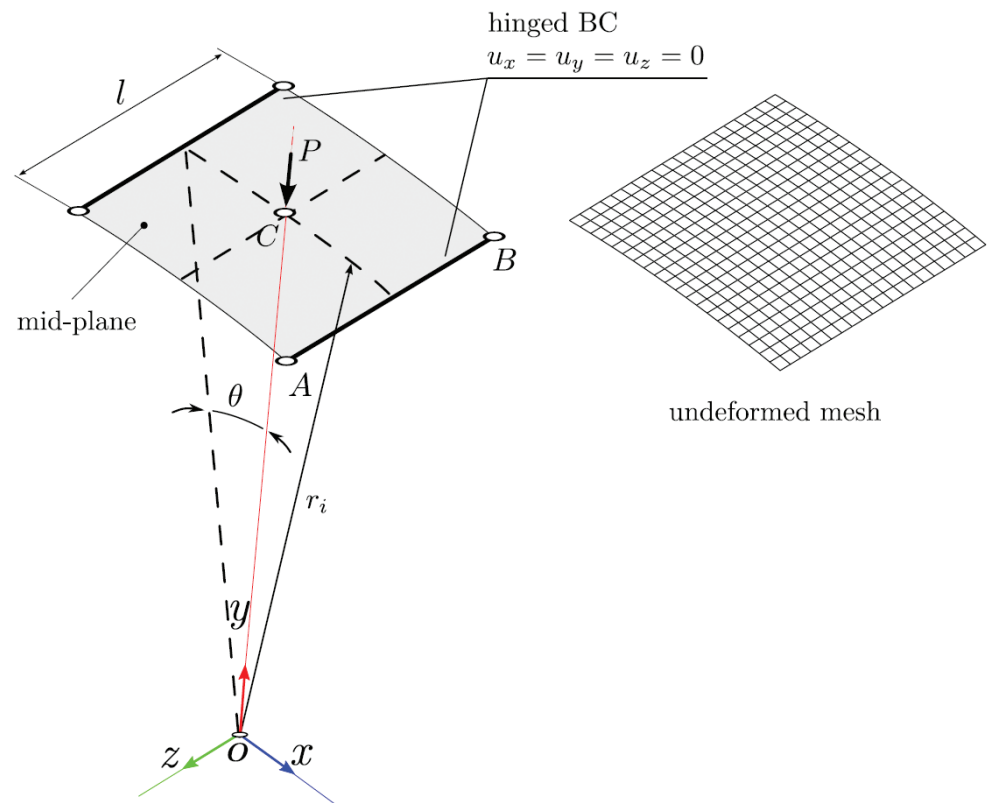

Figure 10. Hinged cylindrical laminate problem §3.2.4.

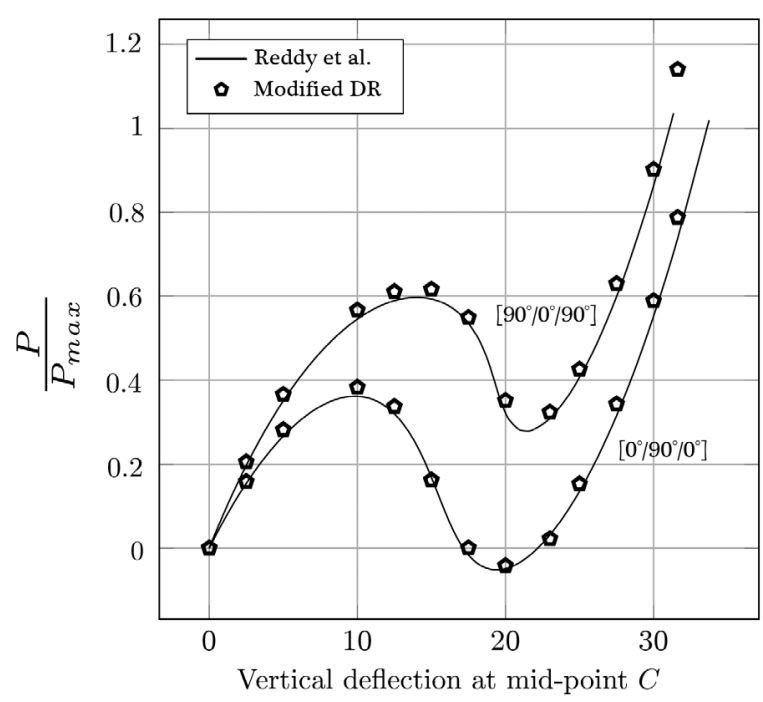

Figure 11. Hinged cylindrical laminate problem: vertical deflection of the mid-point versus relaxed reaction force.

In order to assemble the TFM prototype from flat panels, first $\mathbf{A} 1$ and $\mathbf{A} 2$ are positioned respectively at $\mathbf{A}^{\prime} 1$ and $\mathbf{A}^{\prime} 2$ and are fixed. At the next step, $\mathbf{C} 1$ is positioned at $\mathbf{C}^{\prime} 1$ and fixed and finally $\mathbf{C} 2$ is brought to position $\mathbf{C}^{\prime} 2$ and whole is connected to the base steel profile. (See Fig 14.a)

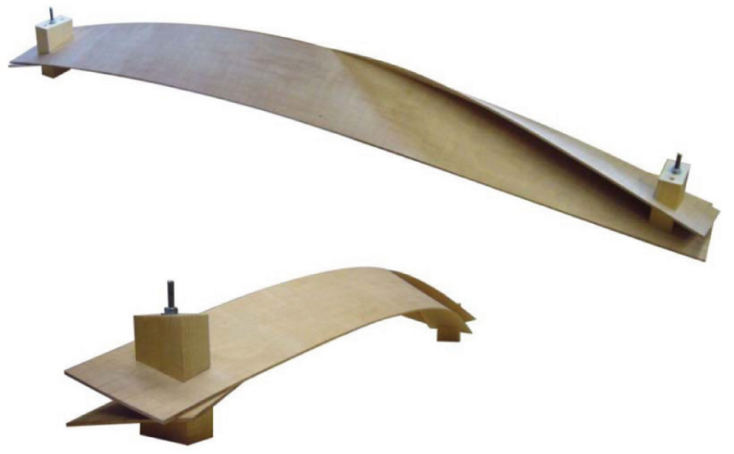

Figure 12. TFM built-in prototype.

(a)
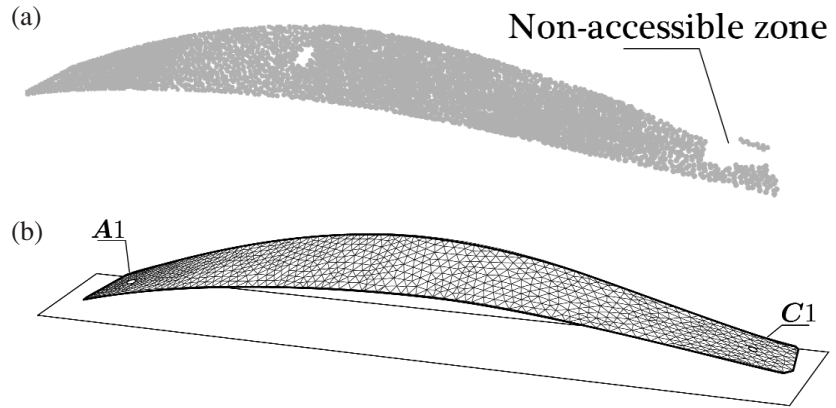

Figure 13. Reconstruction of 3D surface mesh corresponding to the top-surface of Panell (a) the scanned point cloud of the prototype after registration (b) the 3D mesh surface reconstruction of the point cloud (See Fig 14.a). 
(a)

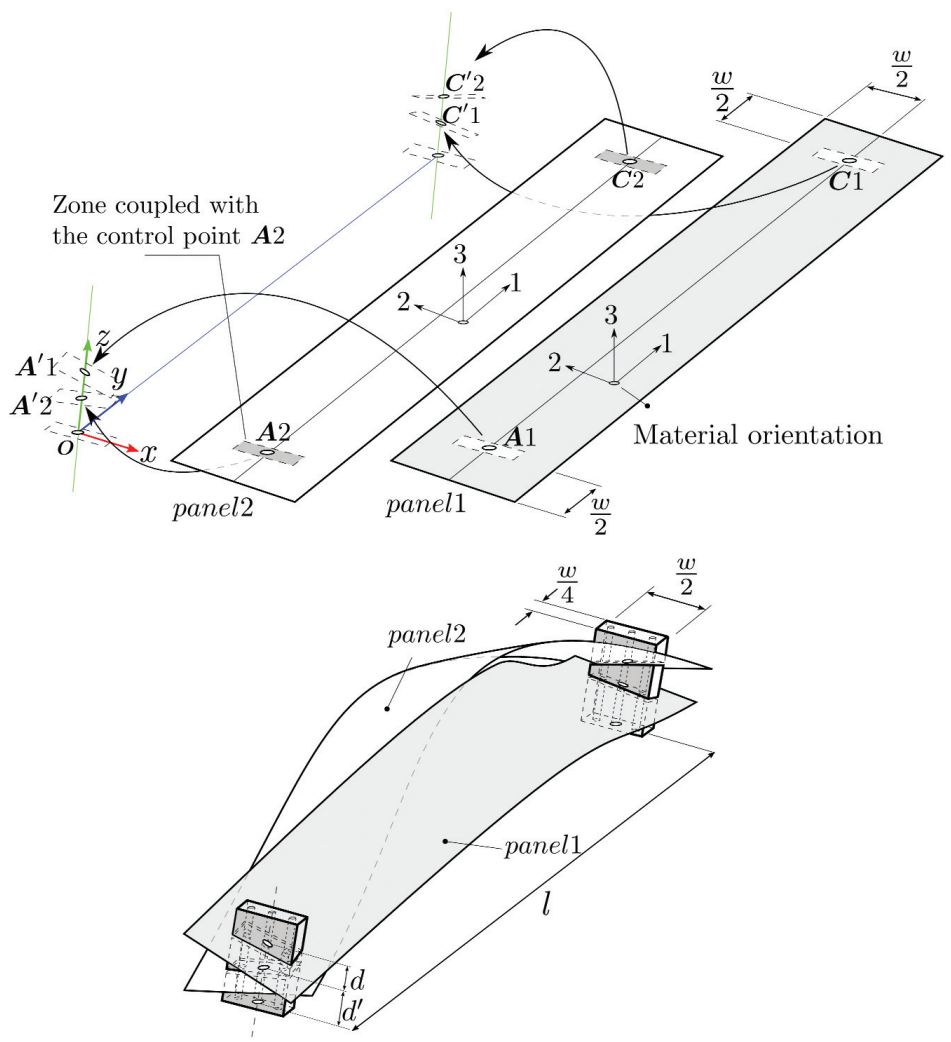

(b)
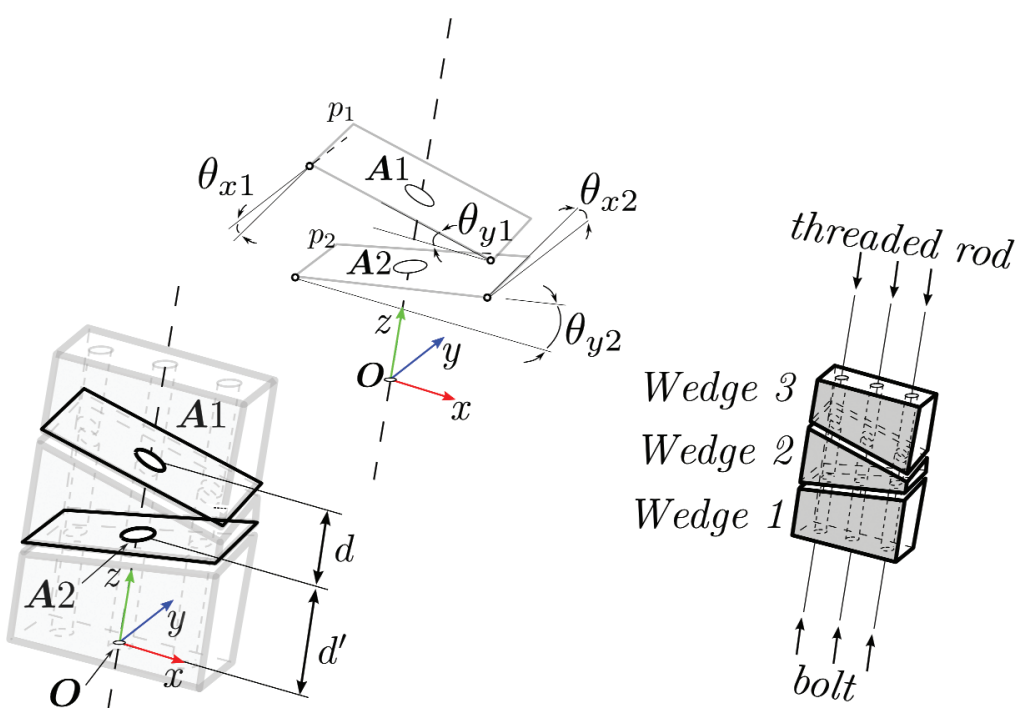

Figure 14. (a) TFM prototype geometry and assembly, (b) Connection relative angles and offset, wedge connection detail.

\subsection{Material properties}

Cross-laminated TeboPly ${ }^{T M}$ Okumé plywood panels, supplied by Thebault (FRANCE) are used to build the prototype. It is a four-ply $[0 / 90]_{s}$ laminate of total thickness of $6.7 \mathrm{~mm}$. Orthotropic material orientation are illustrated in Fig 14.a and homogenized material constants are $E_{1}=4163 \mathrm{Mpa}$, $E_{2}=5088 \mathrm{Mpa}, v_{12}=0.3, G_{12}=552 \mathrm{Mpa}, G_{13}=$ $G_{23}=91 \mathrm{Mpa}$, which correspond to the values obtained from specimens tested in the ambient temperature and humidity.

\subsection{Surface measurement using Laser scanner FARO arm and mesh processing}

In order to compare simulation results and validation, the top surface of the Panell (See Fig 14.a for Panell at RHS) from the prototype is scanned using 3D noncontact scanning technique with the Laser FARO arm 

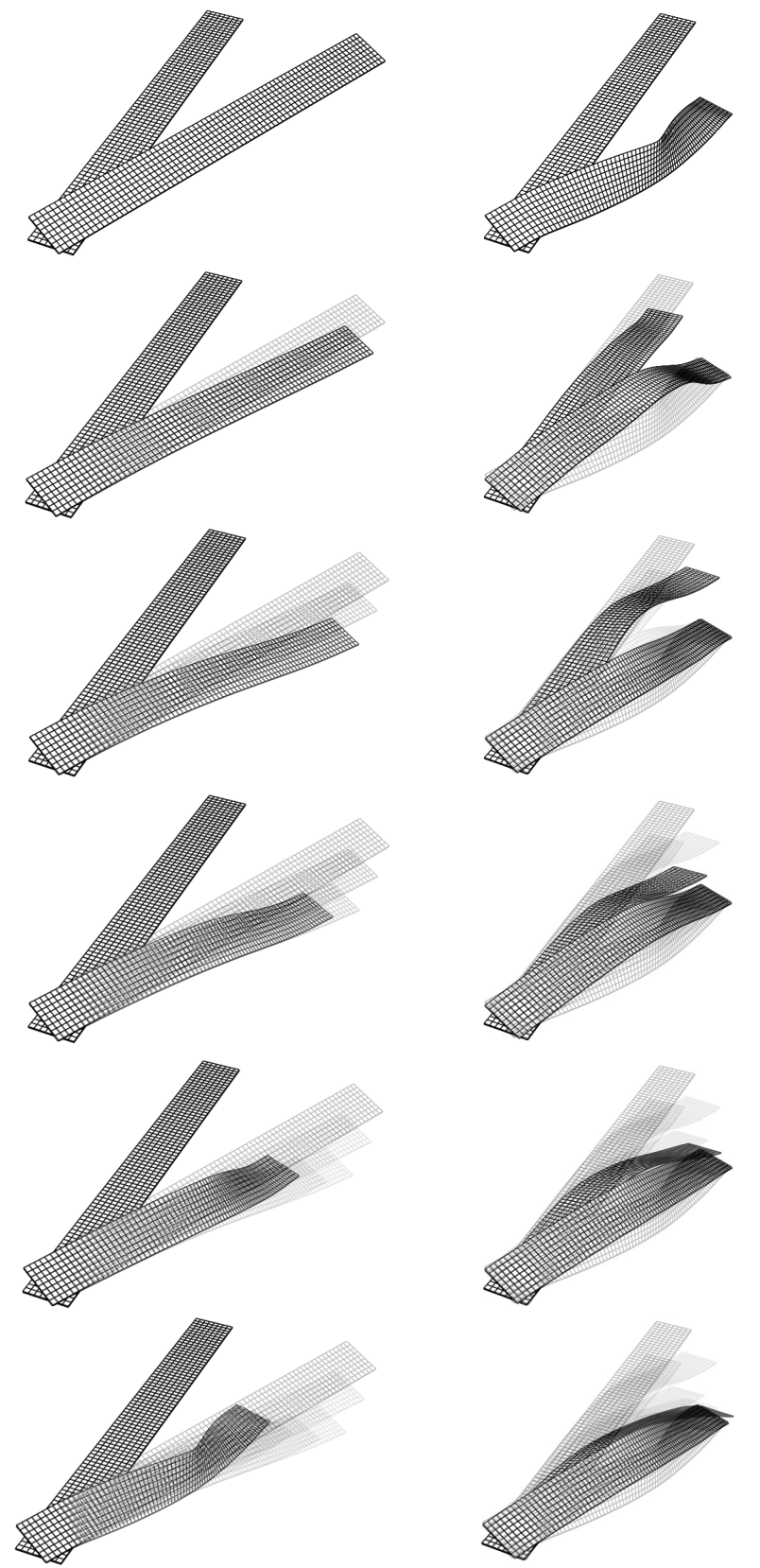

Figure 15. Deformed snapshots for simulation Step1 $(0.0 \leq t \leq$ 2.0 see column (a), view from top to bottom) and Step2 $(2.0 \leq$ $t \leq 4.0$ see column (b) view from top to bottom).

scanner. Regarding the size of the prototype scanning is preformed through six snapshots with sufficient superposition to ease the Registration. However due to the tight over-closure between panels along the connection zone between $\mathbf{C}^{\prime} 1$ and $\mathbf{C}^{\prime} 2$ handle points, reconstructed panel is slightly smaller than the flat initial rectangle.

In every six snapshots, point cloud is "cleaned" to contain only the vertices corresponding to the top surface of Panell, and filtered to merge together all the vertices that are closer than a $10 \mathrm{~mm}$ threshold. Duplicate vertices are also removed. These point clouds are then aligned and merged using the 3D shape
S, Mises

SNEG, $($ fraction $=-1.0$ )

(Avg: $75 \%$ )
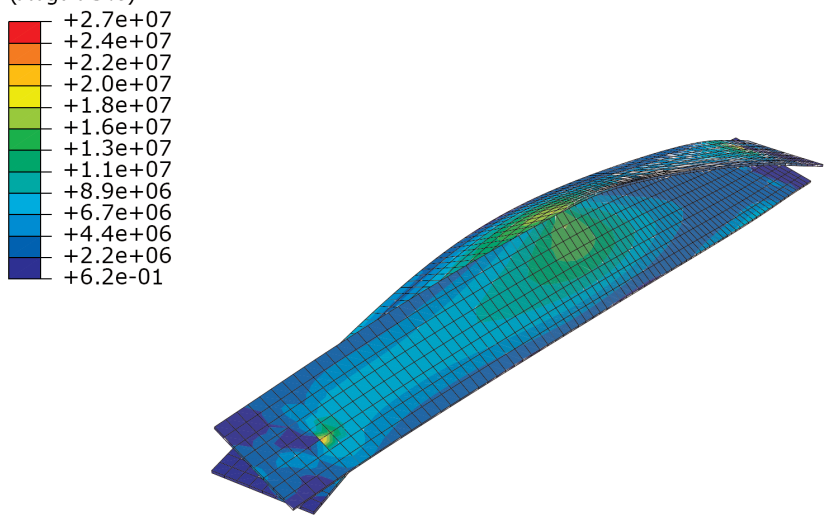

(b)

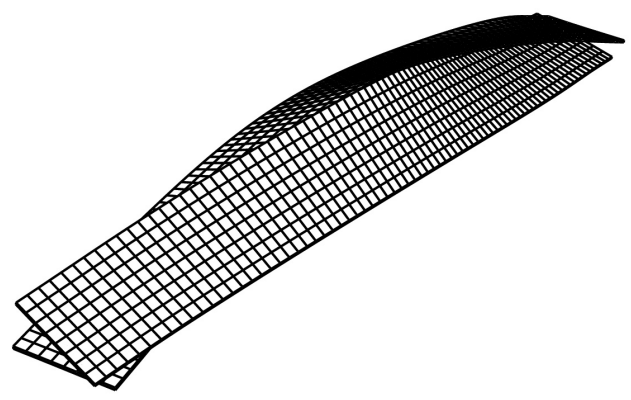

Figure 16. Simulation results at the end of Step3 (a) the VonMises stress on mid-surface panels [Pa] (b) Deformed snapshot for $t=14.0$.

Registration technique based on the ICP algorithm [49]. Later, a 3D triangular surface mesh is reconstructed, to best match the aligned and merged point cloud using the Poisson surface reconstruction technique [50]. The reconstructed mesh, output of Poisson reconstruction, extends beyond the initial point cloud boundary and thus have to be split with polylines corresponding to approximate surfaces edges. This trimmed and reconstructed surface mesh is also re-meshed with uniform $30 \mathrm{~mm}$ mesh size target length, using an isotropic surface-based incremental remeshing technique. [51] This remeshed polygonal 3D mesh (See Fig. 14) is used for comparison with simulation results in $\S 5$.

\subsection{Finite element simulation}

The mid-surfaces for each panel are simulated using quadrilateral shell Finite Element, S4R ABAQUS/ Explicit, with reduced integration scheme and enhanced hourglass control. Each FE node has six degrees of freedom: three translational and three rotational. ABAQUS/Explicit user subroutine 

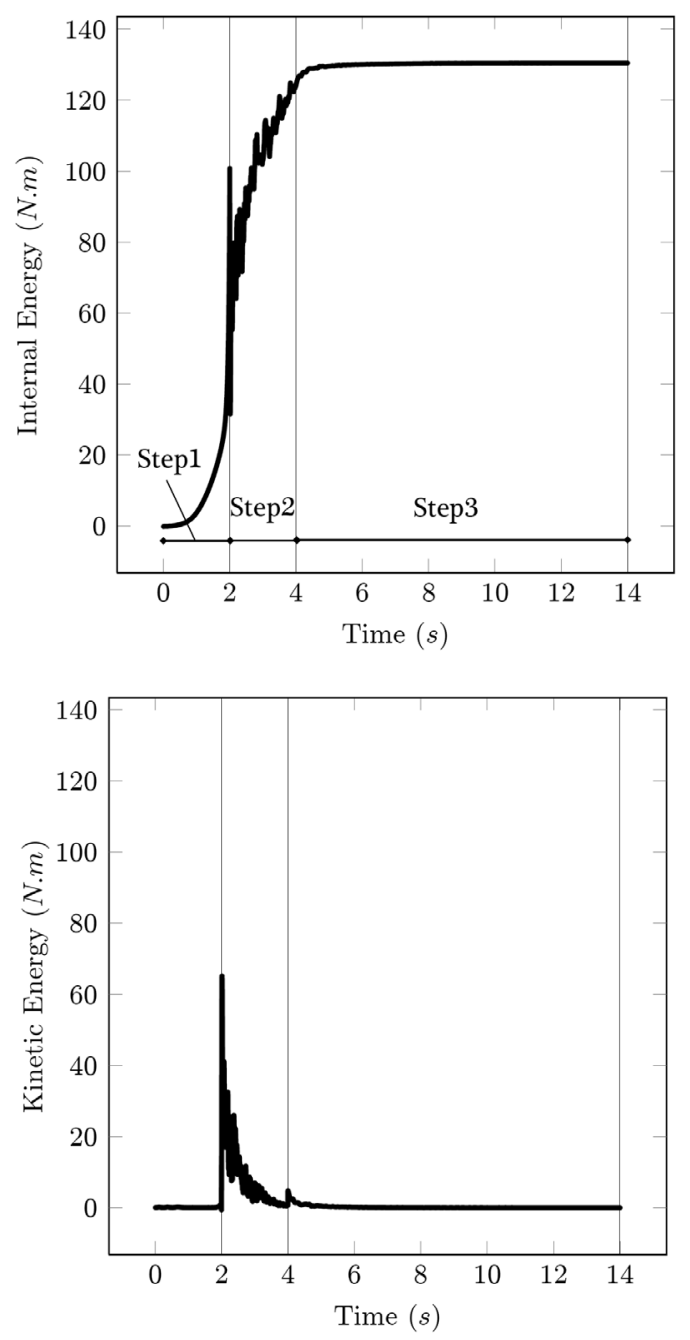

Figure 17. Energy evaluation during the TFM simulation steps.

VUMAT described in $\S 3$ is exploited for this simulation with constant $\gamma=0.9$.

Four fixed points, $\mathbf{A}^{\prime} \mathbf{1}, \mathbf{A}^{\prime} \mathbf{2}, \mathbf{C}^{\prime} \mathbf{1}, \mathbf{A}^{\prime} \mathbf{2}$, are defined in 3D space which represent where corresponding point for each panel should be positioned. On each panel, two nodes are marked as handle points to deform the panel with, A1, C'1 for panell and A2, C2 for panel2. These handle points help to impose Translational/ rotational boundary conditions, forced by connector wedge blocks. A rectangular zone (corresponding to the wedge cross section), is selected per handle point and is rigidly coupled with it over all six degrees of freedom. ABAQUS/Explicit general contact feature is used to handle face and edge contact during all simulation steps. Only non-penetrative hard normal contact is considered and the tangential component is ignored. Form-finding simulation is realized in three steps, described as follows. Since the analysis is pseudo-dynamic, a notion of time length is assigned to each analysis step, but the duration of each step is chosen long enough for imposed boundary conditions to be applied smoothly. (See Figs 14.a and 14.b for notations)

- Step1: $(0.0 \leq t \leq 2.0)$

Panels are positioned with exact relative offset $d, d^{\prime}$ and angle at their left hand side handles $\mathbf{A 1}$, $\mathbf{A 2}$ into fix points $\mathbf{A}^{\prime} \mathbf{1}, \mathbf{A}^{\prime} \mathbf{2}$ respectively. $\theta_{x 1}, \theta_{y 1}$ and $\theta_{x 2}, \theta_{y 2}$ are imposed respectively for $\mathbf{A 1}, \mathbf{A} \mathbf{2}$. All degrees of freedom for these points remain fixed during this first step. Keeping A1, A2 fixed, During a Dynamic/Explicit step, the handle point $\mathbf{C} 1$ is brought to position $\mathbf{C}^{\prime} \mathbf{1}$, and spatial angles $-\theta_{x 2},-\theta_{y 2}$ are imposed. (Wedges are mirrored) Rotational and displacement BCs are imposed simultaneously. The left midpoint of panell, noted as $\mathbf{B 1}$ is also fixed in $\mathrm{x}$ direction order to ease the overlap which will occur in following steps.

- $\quad$ Step2: $(2.0 \leq t \leq 4.0)$

Handle points $\mathbf{A} \mathbf{1}, \mathbf{A} \mathbf{2}$ and $\mathbf{C} \mathbf{1}$ are kept at their deformed position $\mathbf{A}^{\prime} \mathbf{1}, \mathbf{A}^{\prime} \mathbf{2}$ and, $\mathbf{C}^{\prime} \mathbf{1}$ respectively. All degrees of freedom for these three handle points are fixed during this step except $\theta_{z z}$ to let panels reposition respective to each other. The fixed boundary condition for handle point $\mathbf{B} \mathbf{1}$ is also released. Starting the step 2, the point $\mathbf{C 2}$ is brought to position $\mathbf{C}^{\prime} \mathbf{2}$ and spatial angles $-\theta_{x 2}$, $-\theta_{y 1}$ are imposed. During this step panels will collide over the cross section and will reposition themselves into the relaxed configuration.

- Step3: $(4.0 \leq t \leq 14.0)$

Having four handle points at deformed position and exact angles, all degrees of freedom for these four handle points are fixed during this step and the module is left to dissipate extra kinetic energy.

Deformed snapshots extracted with equal time intervals illustrate the deformation of panels during Step1 and Step2. During Step3 Deformed geometry does not considerably vary with the deformed state at the end of Step2 but it is essential to ensure that the system has reached the stationary state to have an accurate stress state. At the end of Step3, deformed coordinates of nodes for the top surface of Panell are extracted and reported for surface reconstruction and comparison with the scanned prototype geometry (See $\S 4.3$ ).

\section{RESULTS AND COMPARISON}

Having in mind our primary objective as form-finding of TFS, we progress with comparison of deformed 3D shapes, issue of modeling and prototype scanning. The main focus here will be on geometrical comparison of 
(a)

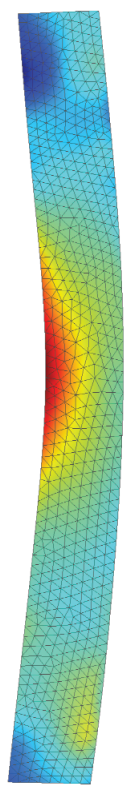

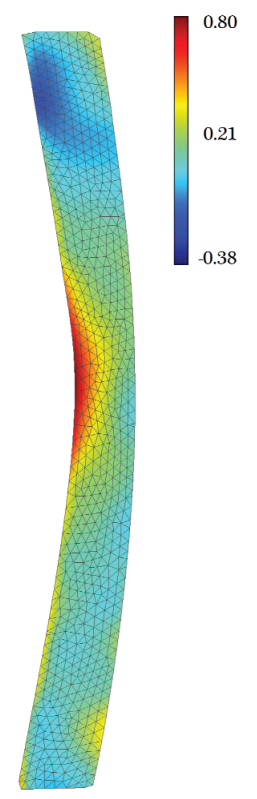

(b)

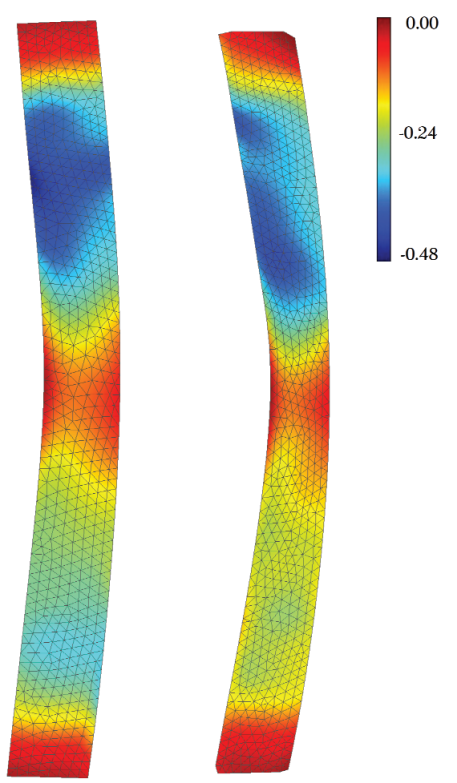

Figure 18. Deformed surface curvature comparison: (a) Mean curvature ( $\left.\mathrm{m}^{-1}\right)$ (b) Gaussian curvature $\left(\mathrm{m}^{-1}\right)$. In each subfigure, simulation extracted surface appears on left and the reconstructed scanned surface of the prototype on right.

these surfaces, rather than the more classical approach of loading the prototype and deformation measurements as tracked in [3].

\subsection{Simulation surface processing}

Deformed coordinates of nodes of the top surface of Panell is extracted from simulation result database for the last frame of the simulation Step3. A triangular $3 \mathrm{D}$ mesh is patched on these vertices and remeshed based on the same technique and criteria described in $\S 4.3$.

\subsection{Surface comparison}

Surfaces are aligned by the help of the ICP Registration algorithm to get the best fit between two 3D meshes. Maximum normal distance of $9 \mathrm{~mm}$ is found between two surfaces. Further inquiry on correspondence between surfaces would be to inspect their local curvature properties, the mean and Gaussian curvature. These local properties on 3D mesh surfaces can be estimated using the Algebraic Point Set Surface (APSS) approximated curvatures of the best fitting spheres to the mesh point set. [52]

Results for theses approximated curvatures are respectively shown in Figs 18.a and 18.b and show a good correlation between numerical and experimental results, for both absolute value and distribution.

The estimated value for the Gaussian curvature takes nonzero values, particularly in surface zones extending between the mid-contact point and the connection wedge, which means that TFM deformed surface is not developable in its precise definition. It also means that the deformation map from the flat panel into the deformed surface of the TFM is not isometric. Meanwhile the absolute value for the Gaussian curvature remains relatively small. The distribution for Gaussian curvature follows the intuition that flat cut wedges impose the planarity (zero Gaussian curvature) on a locally restrained zone at both ends.

The investigation on the mean curvature leads us to important impact of the mid-contact point between panel-edges, which imposes the local flexural deformation at this area. Transversal stability of each panels in TFM assembly, indeed lays on in-plane stiffness of its dual at this mid-contact point. Distribution of both Gaussian and mean curvature are not symmetric which limits the eventual simplifications of the simulation.

\section{CONCLUSION AND FUTURE WORK}

Pseudo dynamic Explicit Finite Element method such as Dynamic Relaxation, can effectively deal with geometrically nonlinear problems of form finding and initial stress prediction for space structures issue of free manipulation of thin shells undergoing finite rotation.

Timber Fabric Structures (TFS) are novel concept structures coming from a reinterpretation of knot and braided structures with thin timber panels and can be generalized into a wide family of space structures where the form and structural behavior are intensely interrelated. 
Mesh processing pipeline (from shape Laser scanning up to 3D mesh surface reconstruction and discrete curvature estimation techniques) seems to represent a promising approach for complex geometry, structural form investigation and may efficiently replace classical loading experiments and reshape the special focus on the deformed shape comparison.

The simulation procedure with geometrically nonlinear shell FE although precise, is expensive and less reactive to design changes. Consequently we are currently working on application of physically based modeling techniques for discrete thin shell and elastic rod simulation, to approach a more interactive finite strip simulator and aim to integrate it into a physically based NURBS/CAD tool for active bending form exploration.

\section{ACKNOWLEDGMENT}

This work was sponsored by the Swiss National Science Foundation (SNSF) under grant No. 20002_137884/1. This support is gratefully acknowledged.

\section{REFERENCES}

[1] Hudert M. Structural timber fabric: applying textile principals in building scale. Ph.D. thesis; IBOIS, EPFL; 2012.

[2] Weinand Y, Hudert M. Timberfabric: Applying textile principles on a building scale. Architectural Design 2010;80(4):102-7.

[3] Sistaninia M, Hudert M, Humbert L, Weinand Y. Experimental and numerical study on structural behavior of a single timber textile module. Engineering Structures 2013;46(0):557-68.

[4] Otter J, Day A. tidal computations. The Engineer 1960;

[5] Day A. An introduction to dynamic relaxation.The engineer 1965;219:218-21.

[6] Otter J, Cassell A, Hobbs R, et al. Dynamic relaxation. In: Inst. Civ.Engrs. Proceedings; vol. 35. 1966, p. 633-56.

[7] Otter J. Computations for pre-stressed concrete reactor pressure vessels using dynamic relaxation. Nuclear Structural Engineering 1965;1(1):61-75.

[8] Frankel SP. Convergence rates of iterative treatments of partial differential equations. Mathematical Tables and Other Aids to Computation 1950;4(30):pp. 65-75.

[9] Frieze P, Hobbs R, Dowling P. Application of dynamic relaxation to the large deflection elasto-plastic analysis of plates. Computers \& Structures 1978;8(2):301-10.

[10] Pica A, Hinton E. Transient and pseudo-transient analysis of Mindlin plates. International Journal for Numerical Methods in Engineering 1980;15(2):189-208.

[11] Pica A, Hinton E. Further developments in transient and pseudo transient analysis of mindlin plates. International Journal for Numerical Methods in Engineering 1981;17(12):1749-61.

[12] Ramesh G, Krishnamoorthy C. Geometrically nonlinear analysis of plates and shallow shells by dynamic relaxation. Computer methods in applied mechanics and engineering 1995;123(1):15-32.
[13] Kommineni J, Kant T. Pseudo-transient large deflection analysis of composite and sandwich shells with a refined theory. Computer methods in applied mechanics and engineering 1995;123(1):1-13.

[14] Ramesh G, Krishnamoorthy C. Postbuckling analysis of structures by dynamic relaxation. International journal for numerical methods in engineering 1993;36(8):1339-64.

[15] Papadrakakis M. Postbuckling analysis of spatial structures by vector iteration methods. Computers \& structures 1981;14(5):393-402.

[16] Alamatian J. Displacementbased methods for calculating the buckling load and tracing the postbuckling regions with dynamic relaxation method. Computers and Structures 2013;114115:84-97.

[17] Barnes M. Form finding and analysis of tension structures by dynamic relaxation. International journal of space structures 1999;14(2):89-104.

[18] Adriaenssens S, Barnes M. Tensegrity spline beam and grid shell structures. Engineering structures 2001;23(1):29-36.

[19] Ye J, Feng R, Zhou S. The modified dynamic relaxation method for the form-finding of membrane structures. Advanced Science Letters 2011;4(810):2845-53.

[20] Bel Hadj Ali N, RhodeBarbarigos L, Smith I. Analysis of clustered tensegrity structures using a modified dynamic relaxation algorithm. International Journal of Solids and Structures 2011;48(5):637-47.

[21] Rodriguez J, Rio G, Cadou J, Troufflard J. Numerical study of dynamic relaxation with kinetic damping applied to inflatable fabric structures with extensions for $3 \mathrm{~d}$ solid element and nonlinear behavior. Thin Walled Structures 2011;49(11):1468-74.

[22] Douthe C, Baverel O. Design of nexorades or reciprocal frame systems with the dynamic relaxation method. Computers \& Structures 2009;87(21):1296-307.

[23] Joldes G, Wittek A, Miller K. An adaptive dynamic relaxation method for solving nonlinear finite element problems. application to brain shift estimation. International journal for numerical methods in biomedical engineering 2011;27(2):173-85.

[24] Brew J, Brotton D. Nonlinear structural analysis by dynamic relaxation. International J. for Numerical Methods in Engineering1971;3(4):463-83.

[25] Wood WL. Note on dynamic relaxation. International Journal for Numerical Methods in Engineering 1971;3(1):145-7.

[26] Papadrakakis M. A method for the automatic evaluation of the dynamic relaxation parameters. Computer methods in applied mechanics and engineering 1981;25(1):35-48.

[27] Underwood P. Dynamic relaxation(in structural transient analysis). Computational methods for transient analysis(A 8429160 1264) Amsterdam, North-Holland, 1983, 1983;:245-65.

[28] Cassell A, Hobbs R. Numerical stability of dynamic relaxation analysis of nonlinear structures. International Journal for Numerical Methods in Engineering 1976;10(6):1407-10.

[29] Alwar R, Rao N, Rao M. An alternative procedure in dynamic relax ation(structural analysis using damped oscillation envelope). Computers \& Structures 1975;5:271-4.

[30] TONG P, TSUI TY. Stability of transient solution of moderately thick plate by finitedifference method. AIAA journal 1971;9(10):2062-3. 
[31] Rezaiee-Pajand M, Kadkhodayan M, Alamatian J. Timestep selection for dynamic relaxation method. Mechanics Based Design of Structures and Machines 2012;40(1):42-72.

[32] Alamatian J. A new formulation for fictitious mass of the dynamic relaxation method with kinetic damping. Computers and Structures 2012;9091(1):42-54.

[33] Rezaiee-Pajand M, Sarafrazi S, Rezaiee H. Efficiency of dynamic re laxation methods in nonlinear analysis of truss and frame structures. Computers and Structures 2012;112113:295-310.

[34] Rezaiee-Pajand M, Kadkhodayan M, Alamatian J, Zhang L. A new method of fictitious viscous damping determination for the dynamic relaxation method. Computers and Structures 2011;89(910):783-94.

[35] Rezaiee-Pajand M, Sarafrazi S. Nonlinear dynamic structural analysis using dynamic relaxation with zero damping. Computers and Structures 2011;89(1314): 1274-85.

[36] Rushton K. Large deflection of variable thickness plates. International Journal of Mechanical Sciences 1968; 10(9):723 -35.

[37] Chung W, Cho J, Belytschko T. On the dynamic effects of explicit fem in sheet metal forming analysis. Engineering Computations: Int $\mathrm{J}$ for ComputerAided Engineering 1998;15(6):750-76.

[38] Cassell A, Kinsey P, Sefton D. Cylindrical shell analysis by dynamic relaxation. In: Inst. Civ. Engrs. Proceedings; vol. 39. 1968, p. 75-84.

[39] Lynch R, Kelsey S, Saxe H. The application of dynamic relaxation to the finite element method of structural analysis. Tech. Rep.; Technical report No. THEMISUND681 (University of Notre Dame); 1968.

[40] Hughes T. Analysis of transient algorithms with particular reference to stability behavior. Computational methods for transient analysis(A 8429160 1264) Amsterdam, North-Holland, 1983, $1983 ;: 67-155$.

[41] Belytschko T. An overview of semi-discretization and time integration procedures. Computational methods for transient analysis(A 8429160 1264) Amsterdam, NorthHolland, 1983, 1983;:1-65.

[42] Courant R, Friedrichs K, Lewy H. Über die partiellen differenzengleichungen der mathematischen physik. Mathematische Annalen 1928;100(1):32-74.

[43] Tabiei A, Tanov R. A nonlinear higher order shear deformation shell element for dynamic explicit analysis:: Part i. formulation and finite element equations. Finite Elements in Analysis and Design 2000;36(1):17-37.

[44] Sze K, Liu X, Lo S. Popular benchmark problems for geometric nonlinear analysis of shells. Finite elements in analysis and design 2004;40(11):1551-69.

[45] Arciniega R, Reddy J. Tensor-based finite element formulation for geometrically nonlinear analysis of shell structures. Computer Methods in Applied Mechanics and Engineering 2007;196(4):1048-73.

[46] Buechter N, Ramm E. Shell theory versus degeneration a comparison in large rotation finite element analysis. International Journal for Numerical Methods in Engineering 1992;34(1):39-59.

[47] Simo J, Fox D, Rifai M. On a stress resultant geometrically exact shell model. part iii: Computational aspects of the nonlinear theory. Computer Methods in Applied Mechanics and Engineering 1990;79(1):21-70.

[48] Jiang L, Chernuka M. A simple four-noded co-rotational shell element for arbitrarily large rotations. Computers and Structures 1994;53(5):1123 -32.

[49] Besl P, McKay N. A method for registration of 3d shapes. IEEE Trans actions on pattern analysis and machine intelligence 1992;14(2):239-56.

[50] Kazhdan M, Bolitho M, Hoppe H. Poisson surface reconstruction. In: Proceedings of the fourth Eurographics symposium on Geometry processing. Eurographics Association; 2006, p. 61-70.

[51] Botsch M, Kobbelt L. A remeshing approach to multiresolution modeling. In: Proceedings of the 2004 Eurographics/ACM SIGGRAPH symposium on geometry processing. ACM; 2004, p. 185-92.

[52] Guennebaud G, Gross M. Algebraic point set surfaces. ACM TransGraph 2007;26(3). 